\title{
A geometric obstruction to the contact type property
}

\section{Kai Cieliebak}

Harvard University, Department of Mathematics, Science Center 322, 1 Oxford Street, Cambridge, MA 02138, USA (e-mail: cielieba@math.harvard.edu)

\section{Introduction}

Prompted by the existence results for periodic orbits on energy surfaces, in 1979 A. Weinstein introduced the following concept ([We2]): Let $(M, \omega)$ be a symplectic manifold, i.e. a manifold $M$ of even dimension $2 n$ with a nondegenerate closed 2-form $\omega$. A hypersurface $S \subset M$ (throughout this paper all hypersurfaces are assumed to be smooth without boundary) is said to be of contact type if there exists a 1-form $\lambda$ on $S$ such that

(i) $d \lambda=\left.\omega\right|_{S}$, and

(ii) $\lambda \wedge(d \lambda)^{n-1}$ is a volume form on $S$.

This condition has proved extremely fruitful, mainly for the following two properties of a hypersurface $S$ of contact type:

1. (Stability): There exists a diffeomorphism $\phi:[-\epsilon, \epsilon] \times S \rightarrow W$ onto a tubular neighborhood $W$ of $S$ in $M, \phi(\{0\} \times S)=S$, such that all hypersurfaces $S_{\rho}=\phi(\{\rho\} \times S)$ are conformally symplectomorphic to $S$, i.e. $\left(S_{\rho}, \omega\right)$ is symplectomorphic to $(S, r \omega)$ for some constant $r(\rho)>0$. In particular, the characteristic foliations on all $S_{\rho}$ are conjugate. Here the characteristic foliation of a hypersurface $S \subset(M, \omega)$ is the 1-dimensional foliation consisting of the integral curves of the line bundle $\operatorname{ker}\left(\left.\omega\right|_{S}\right) \rightarrow S$. Its leaves are called characteristics.

2. (J-convexity): Suppose that the hypersurface $S$ is cooriented by a normal vector field $\nu$. Then in a neighborhood of $S$ we can speak of the interior and exterior, defined by the condition that $\nu$ points to the exterior of $S$. We say that $(S, \nu)$ is $\omega$-convex if there exists a 1 -form $\lambda$ on $S$ satisfying (i) and (ii) such that $\lambda \wedge(d \lambda)^{n-1}$ is a positive multiple of $\left.i_{\nu}\left(\omega^{n}\right)\right|_{S}$, where $i_{\nu}$ is the contraction with $\nu$. In this case there exists a compatible almost 
complex structure $J$ on $(M, \omega)$, i.e. an almost complex structure $J$ such that $\omega(\cdot, J \cdot)$ is a Riemannian metric, for which $S$ is $J$-convex in the sense that no J-holomorphic curve can touch $S$ from the interior.

Given a cooriented hypersurface $(S, \nu) \subset(M, \omega)$ it is in general difficult to decide whether it is of contact type. An obvious necessary condition arises from the closed characteristics. Suppose that $\left.\omega\right|_{S}$ is exact. Then to a closed characteristic $x: S^{1}:=\mathbf{R} / \mathbf{Z} \rightarrow S$ which is homologically trivial, $[x]=0 \in H_{1}(S)$, and oriented positively, i.e. such that $\omega(\dot{x}, \nu)>0$, we associate its action

$$
A(x):=-\int_{S^{1}} x^{*} \alpha
$$

where $\alpha$ is any 1-form on $S$ with $d \alpha=\left.\omega\right|_{S}$. This definition does not depend on the choice of $\alpha$. If $(S, \nu)$ is $\omega$-convex with contact form $\lambda$, then $A(x)=-\int_{x} \lambda>0$ for all homologically trivial closed characteristics $x$ (similarly $<0$ if $S$ is $\omega$-concave).

More generally, let us fix a vector field $X$ generating $\operatorname{ker}\left(\left.\omega\right|_{S}\right)$ with $\omega(X, \nu)>$ 0 . Then every finite Borel measure $\mu$ on $S$ acts on 1-forms $\beta$ on $S$ via

$$
\langle\mu, \beta\rangle:=\int_{S} \beta(X) d \mu .
$$

We say that $\mu$ is exact as a current if $\langle\mu, \beta\rangle=0$ for all closed 1-forms $\beta$. Then we have the following criterion due to D.Sullivan and D.McDuff ([Su], [McD], see also [EG]): A closed hypersurface $S \subset(M, \omega)$ with $\left.\omega\right|_{S}=d \alpha$ is of contact type if and only if there exists a constant $c$ such that $\langle\mu, \alpha\rangle \geq c \cdot \mu(S)$ for every finite $X$-invariant Borel measure $\mu$ on $S$ which is exact as a current.

Notice that Sullivan's criterion, like the original definition, depends on the characteristic foliation and thus on the $C^{1}$-type of the hypersurface. Under $C^{0}$-small perturbations the characteristic foliation may change drastically (see e.g. [Ci]). We will now give a geometric obstruction to the contact type property which is stable under $C^{0}$-small perturbations.

Consider the space $\mathbf{R}^{2 n}$ with coordinates $\left(x_{1}, \ldots, x_{n}, y_{1}, \ldots, y_{n}\right)$ and the standard symplectic form $\omega_{2 n}:=d \lambda_{2 n}$, where

$$
\lambda_{2 n}:=\frac{1}{2} \sum_{j=1}^{n}\left(x_{j} d y_{j}-y_{j} d x_{j}\right) .
$$

Define the action of a loop $x: S^{1} \rightarrow \mathbf{R}^{2 n}$ as

$$
A(x):=-\int_{S^{1}} x^{*} \lambda_{2 n} .
$$



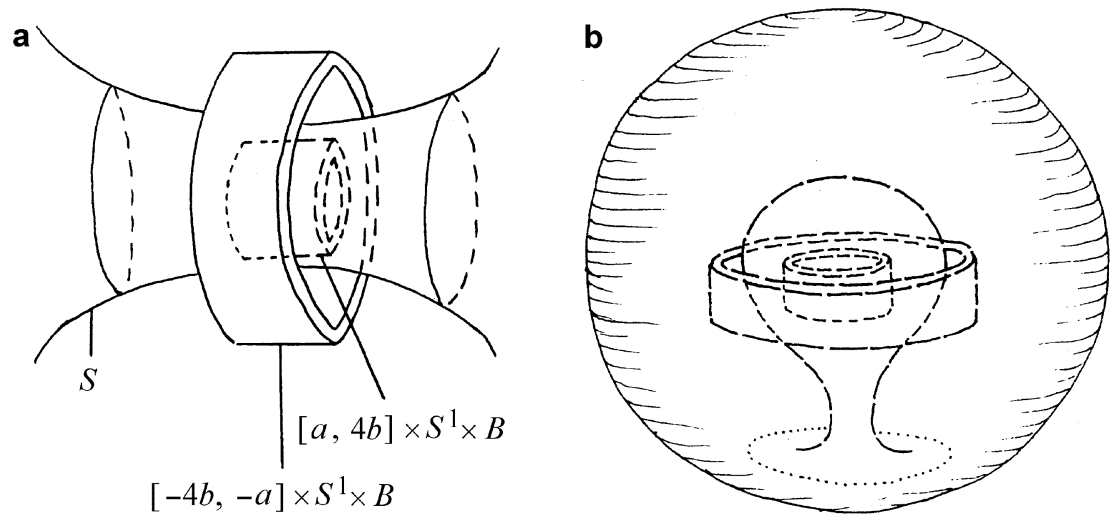

Fig. 1.

For a connected compact hypersurface $S \subset \mathbf{R}^{2 n}$ (without boundary) we denote by $B(S)$ the bounded and by $U(S)$ the unbounded component of $\mathbf{R}^{2 n} \backslash S$. By $B^{k}(r)$ we denote the closed ball around zero in $\mathbf{R}^{k}$ of radius $r$.

A hypersurface $S$ in a symplectic manifold $(M, \omega)$ is said to be of $r e$ stricted contact type if there exists a 1-form $\lambda$ on $S$ satisfying (i) and (ii) which extends to a 1 -form $\bar{\lambda}$ on $M$ with $d \bar{\lambda}=\omega$. Notice that if $\omega$ is exact and $H^{1}(S)=\{0\}$, then 'contact type' and 'restricted contact type' are equivalent conditions.

Theorem 1. For $n \geq 2$ there exist numbers $0<a \leq b$ and an embedding $f:[-4 b, 4 b] \times S^{1} \times B^{2 n-2}(4 b) \hookrightarrow \mathbf{R}^{2 n}$ with the following property:

If $S \subset \mathbf{R}^{2 n}$ is a connected compact hypersurface such that

$$
\begin{aligned}
& f\left([-4 b,-a] \times S^{1} \times B^{2 n-2}(4 b)\right) \subset B(S), \\
& f\left([a, 4 b] \times S^{1} \times B^{2 n-2}(4 b)\right) \subset U(S),
\end{aligned}
$$

then $S$ is not of restricted contact type.

Figure 1 shows two examples of hypersurfaces satisfying the hypotheses of Theorem 1. In Fig. 1.a) the hypersurface $S$ closes up to a hypersurface of type $S^{1} \times S^{2 n-2}$ surrounding the larger ring $[-4 b,-a] \times S^{1} \times B^{2 n-2}(4 b)$.

Remark. The embedding $f$ is symplectic with respect to a twisted symplectic structure on $[-4 b, 4 b] \times S^{1} \times B^{2 n-2}(4 b)$ in which the circles $\{\rho\} \times$ $S^{1} \times\{0\}^{2 n-2}$ are nondegenerate closed characteristics on the hypersurfaces $\{\rho\} \times S^{1} \times B^{2 n-2}(4 b)$ (see Sect. 3).

The Hausdorff metric on the space of all closed bounded subsets of a metric space $(X, d)$ is defined as

$$
d_{H}(A, B):=\max \left\{\sup _{a \in A} d(a, B), \sup _{b \in B} d(b, A)\right\} .
$$


It is easy to see that every compact hypersurface $S \subset \mathbf{R}^{2 n}$ can be approximated in the Hausdorff metric by compact hypersurfaces of restricted contact type: Approximate $S$ in $d_{H}$ by an embedded closed curve $L \subset \mathbf{R}^{2 n} \backslash S$ of positive action. Then a small tubular neighborhood of $L$ is of restricted contact type and $d_{H}$-close to $S$.

However, this example seems quite artificial. It can be ruled out by a simple topological hypothesis. Let $H y p^{0}\left(\mathbf{R}^{2 n}\right)$ be the space of all connected compact hypersurfaces $S \subset \mathbf{R}^{2 n}$ such that 0 lies in the bounded component of $\mathbf{R}^{2 n} \backslash S$. Then we have

Corollary 1. A hypersurface $S \in H y p^{0}\left(\mathbf{R}^{2 n}\right)$ as in Theorem 1 cannot be approximated in the Hausdorff metric by hypersurfaces in $H_{y p}{ }^{0}\left(\mathbf{R}^{2 n}\right)$ of restricted contact type.

We also get a different criterion in terms of closed characteristics. For a closed characteristic $x$ on a hypersurface $S$ consider its linear Poincaré $m a p$, i.e. the linearization of the Poincaré return map on a transverse section to $x$. The characteristic $x$ is called nondegenerate if 1 is not an eigenvalue of the linear Poincare map. It is called linearly stable (cf. [GL]) if its linear Poincaré map is symplectically conjugate to a diagonal matrix $\operatorname{diag}\left(e^{i \alpha_{1}}, \ldots, e^{i \alpha_{n-1}}\right) \in \mathbf{C}^{(n-1) \times(n-1)}$ with $\alpha_{1}, \ldots, \alpha_{n-1} \in \mathbf{R}$.

Corollary 2. Suppose that a hypersurface $S \in H y p^{0}\left(\mathbf{R}^{2 n}\right)$ carries a nondegenerate linearly stable closed characteristic $x$ of negative action. Then $S$ cannot be approximated in the Hausdorff metric by hypersurfaces in $H_{y p}{ }^{0}\left(\mathbf{R}^{2 n}\right)$ of restricted contact type.

Remark. In other words, a necessary condition for $S$ to be approximable by hypersurfaces of restricted contact type is the absence of nondegenerate linearly stable closed characteristics of negative action. This condition is definitively not sufficient. Combining Corollary 2 with the construction in [Ci] we find hypersurfaces $S \in H y p^{0}\left(\mathbf{R}^{2 n}\right)$ which carry no closed characteristic of negative action, but still cannot be approximated by hypersurfaces of restricted contact type.

Following Y. Eliashberg and M. Gromov ([EG]), let us call an open (i.e. without compact connected components) symplectic manifold $(M, \omega)$ convexly exhaustible if it admits an exhaustion $U_{1} \subset U_{2} \subset \ldots \subset M$, $\cup_{i \in \mathbf{N}} U_{i}=M$, by compact subsets $U_{i}$ with smooth $\omega$-convex boundaries (cooriented by outward pointing normal vector fields). We call $(M, \omega)$ exact convexly exhaustible if the boundaries $\partial U_{i}$ are exact convex, i.e. the positive contact forms on $\partial U_{i}$ extend to $M$ as primitives of $\omega$. It was shown in [EG] that the complement of a small closed ball in a symplectic manifold is not convexly exhaustible. However, this cannot be applied, e.g., to find 
nonconvex symplectic structures on the open $2 n$-ball. The existence of such structures follows from the following corollary, choosing a hypersurface $S$ which bounds a ball.

Corollary 3. For a hypersurface $S$ as in Corollary 1 or 2, the bounded component of $\mathbf{R}^{2 n} \backslash S$ is not exact convexly exhaustible.

More generally, we have

Corollary 4. Every open manifold $M$ of dimension $2 n \geq 4$ which admits a symplectic structure also admits a symplectic structure which is not exact convexly exhaustible.

Remarks. 1. Corollary 3 answers a question in [EG]: The exact convex exhaustibility of the interior of a domain $\Omega \subset \mathbf{R}^{2 n}$ with smooth boundary $\partial \Omega$ implies certain convexity of $\partial \Omega$. For example, $\partial \Omega$ cannot have a shape as described in Theorem 1 and Fig. 1.

2. The additional question in [EG] whether the actions of all invariant measures on $\partial \Omega$ are nonnegative if the interior is convexly exhaustible must be answered in the negative: By the construction in [Ci] one can always introduce closed characteristics on $\partial \Omega$ with negative action without changing symplectically the interior of $\Omega$.

This paper is organized as follows:

In Sect. 2 we will reduce Theorem 1 to a statement about symplectic homology of hypersurfaces in $\mathbf{R}^{2 n}$ (Theorem 2).

Theorem 2 depends on a version of the Monotonicity Lemma for pseudoholomorphic curves with a Hamiltonian term which will be proved in Sect. 3 . In Sect. 4 we will prove Theorem 2 as well as Corollaries 1-4.

Acknowledgements. I thank L. Moatty whose discontent at the results of [Ci] motivated the present article. I thank D. Nikolenkov for carefully checking the proofs and improving the presentation, E. Zehnder for useful comments, and S. Kim for Fig. 1.b).

\section{Localization of symplectic homology and proof of Theorem 1}

We will first define the version of symplectic homology which we will use to prove Theorem 1. For details we refer the reader to $[\mathrm{FH}]$, [FHW], [CFH] and $[\mathrm{CFHW}$. We shall describe the construction on the standard symplectic space $\left(\mathbf{R}^{2 n}, \omega_{2 n}=d \lambda_{2 n}\right)$, although it works for any exact symplectic manifold which is convexly exhaustible.

Consider a 1-periodic time-dependent Hamiltonian $H: S^{1} \times \mathbf{R}^{2 n} \rightarrow \mathbf{R}$ such that

$$
H(t, x)=0 \quad \text { for }|x| \text { large }
$$


To a smooth loop $x: S^{1} \rightarrow \mathbf{R}^{2 n}$ we associate its Hamiltonian action

$$
A_{H}(x):=-\int_{0}^{1} x^{*} \lambda_{2 n}-\int_{0}^{1} H(t, x(t)) d t .
$$

Critical points of $A_{H}$ are precisely the 1-periodic solutions $x: S^{1} \rightarrow \mathbf{R}^{2 n}$ of

$$
\dot{x}(t)=X_{H}(t, x(t)),
$$

where $X_{H}$ is the Hamiltonian vector field defined by

$$
d H_{t}(x)=\omega_{2 n}\left(X_{H}(t, x), \cdot\right),
$$

and $H_{t}=H(t, \cdot)$.

Fix an interval $[a, b)$ not containing 0 . We call $H$ a regular Hamiltonian if all 1-periodic solutions of $\dot{x}=X_{H}(t, x)$ with $A_{H}(x) \in[a, b)$ are nondegenerate. Observe that the degenerate constant solutions in the region $\{H \equiv 0\}$ have action $0 \notin[a, b)$.

More generally, we will consider Hamiltonians $H: \mathbf{R} \times S^{1} \times \mathbf{R}^{2 n} \rightarrow \mathbf{R}$ satisfying

$$
\begin{gathered}
\frac{\partial H}{\partial s}(s, t, x) \leq 0 \text { for all }(s, t, x), \\
H(s, t, x)= \begin{cases}H_{1}(t, x) & \text { for } s \leq-s_{0} \\
H_{2}(t, x) & \text { for } s \geq s_{0},\end{cases} \\
H(s, t, x)=0 \text { for }|x| \text { large. }
\end{gathered}
$$

Let $J$ be an $(s, t)$-dependent almost complex structure on $\mathbf{R}^{2 n}$ such that

$$
\begin{gathered}
\omega_{2 n}(J(s, t) \cdot, \cdot) \text { is a Riemannian metric for all }(s, t), \\
J(s, t, x)= \begin{cases}J_{1}(t, x) & \text { for } s \leq-s_{0} \\
J_{2}(t, x) & \text { for } s \geq s_{0},\end{cases} \\
J(s, t, x)=i \text { for }|x| \text { large. }
\end{gathered}
$$

Consider smooth maps $\hat{u}: Z=\mathbf{R} \times S^{1} \rightarrow \mathbf{R}^{2 n}$ satisfying

$$
\begin{gathered}
\hat{u}_{s}+J(s, t, \hat{u}) \hat{u}_{t}+\nabla_{J_{s, t}} H(s, t, \hat{u})=0, \\
\hat{u}(s, \cdot) \longrightarrow \begin{cases}x_{1} & \text { as } s \rightarrow-\infty ; \\
x_{2} & \text { as } s \rightarrow+\infty,\end{cases}
\end{gathered}
$$

where $x_{i}$ are 1-periodic solutions of $\dot{x}_{i}(t)=X_{H_{i}}\left(t, x_{i}(t)\right)$, and $\nabla_{J_{s, t}}$ denotes the gradient with respect to the metric $\omega_{2 n}(J(s, t) \cdot, \cdot)$. 
An $s$-independent pair $(H, J)$ satisfying (H1-3) and (J1-3) is called a regular pair if $H$ is a regular Hamiltonian, and 0 is a regular value of the Fredholm operator

$$
\hat{u} \mapsto \hat{u}_{s}+J(t, \hat{u}) \hat{u}_{t}+\nabla_{J_{t}} H(t, \hat{u})
$$

defined on maps $\hat{u}$ satisfying (u2) with $A_{H}\left(x_{i}\right) \in[a, b)$. An $s$-dependent pair $(H, J)$ satisfying $(\mathrm{H} 1-3)$ and $(\mathrm{J} 1-3)$ is called a regular monotone homotopy between the regular pairs $\left(H_{1}, J_{1}\right)$ and $\left(H_{2}, J_{2}\right)$ if 0 is a regular value of the Fredholm operator

$$
\hat{u} \mapsto \hat{u}_{s}+J(s, t, \hat{u}) \hat{u}_{t}+\nabla_{J_{s, t}} H(s, t, \hat{u})
$$

defined on maps $\hat{u}$ satisfying (u2) with $A_{H_{i}}\left(x_{i}\right) \in[a, b)$. In these cases the spaces

$$
\mathcal{M}\left(x_{1}, x_{2}, H, J\right)
$$

of solutions of $(u 1-2)$ are finite dimensional manifolds.

For a regular pair $(H, J)$ consider the finite-dimensional vector space over $\mathbf{Z}_{2}=\mathbf{Z} / 2 \mathbf{Z}$

$$
\begin{aligned}
C^{[a, b)}(H, J):= & \left\{\sum \alpha_{i} x_{i} \mid \alpha_{i} \in \mathbf{Z}_{2}, x_{i}\right. \text { 1-periodic solutions of } \\
& \left.\dot{x}_{i}=X_{H}\left(t, x_{i}\right) \text { with } A_{H}\left(x_{i}\right) \in[a, b)\right\} .
\end{aligned}
$$

We define a linear operator

$$
\begin{gathered}
\partial: C^{[a, b)}(H, J) \rightarrow C^{[a, b)}(H, J), \\
\partial x:=\sum_{y}\langle x, y\rangle_{1} \cdot y,
\end{gathered}
$$

where $\langle x, y\rangle_{1}$ is the number mod 2 of 1 -dimensional components of $\mathcal{M}(x, y$, $H, J)$. The operator $\partial$ satisfies $\partial^{2}=0$. The homology group

$$
F H^{[a, b)}(H):=\operatorname{ker}(\partial) / \operatorname{im}(\partial)
$$

is called the Floer homology in the action interval $[a, b)$. It is independent of $J$, but it does depend on $H$, as we will describe now.

To a regular monotone homotopy $(H, J)$ between regular pairs $\left(H_{1}, J_{1}\right)$, $\left(H_{2}, J_{2}\right)$ we associate a linear map

$$
\begin{gathered}
\sigma(H, J): C^{[a, b)}\left(H_{1}, J_{1}\right) \rightarrow C^{[a, b)}\left(H_{2}, J_{2}\right), \\
\sigma(H, J) x:=\sum_{y}\langle x, y\rangle_{0} \cdot y,
\end{gathered}
$$

where $\langle x, y\rangle_{0}$ denotes the number mod 2 of 0 -dimensional components of $\mathcal{M}(x, y, H, J)$. It turns out that $\sigma(H, J)$ is a chain map. The induced map 
on Floer homology does not depend on the choice of $(H, J)$. We denote it by

$$
\sigma\left(H_{1}, H_{2}\right): F H^{[a, b)}\left(H_{1}\right) \rightarrow F H^{[a, b)}\left(H_{2}\right) .
$$

For 3 regular Hamiltonians $H_{1} \geq H_{2} \geq H_{3}$ the corresponding maps satisfy the composition law

$$
\sigma\left(H_{2}, H_{3}\right) \circ \sigma\left(H_{1}, H_{2}\right)=\sigma\left(H_{1}, H_{3}\right) .
$$

Next let a compact cooriented hypersurface $S \subset \mathbf{R}^{2 n}$ be given. We do not require $S$ to be connected, so $\mathbf{R}^{2 n} \backslash S$ may have more than two connected components. However, we suppose that $\mathbf{R}^{2 n}=B(S) \cup U(S)$, where $B(S)$, $U(S)$ are (not necessarily connected) components, $B(S)$ is bounded, and $U(S)$ is unbounded. Moreover, we assume that the coorientation of $S$ is defined by a normal vector field $\nu$ which points everywhere into $U(S)$.

Fix a number $r>0$. We call a Hamiltonian $H(s, t, x)$ adapted to $(S, r)$ if it satisfies (H1-3) and

$$
\begin{aligned}
H(s, t, x) & =0 \text { for } x \text { outside some compact subset of } B(S), \\
& -r<H(s, t, x) \leq 0 \text { for all }(s, t, x)
\end{aligned}
$$

(see Fig. 2). The set $A d_{\text {reg }}(S, r)$ of $s$-independent regular Hamiltonians adapted to $(S, r)$ is a partially ordered set via

$$
H_{1} \geq H_{2}: \Longleftrightarrow H_{1}(t, x) \geq H_{2}(t, x) \text { for all }(t, x) .
$$

This partial ordering and the homomorphisms $\sigma\left(H_{1}, H_{2}\right)$ turn the set of Floer homology groups

$$
\left(H F^{[a, b)}(H)\right)_{H \in A d_{r e g}(S, r)}
$$

into a directed system. We define the symplectic homology of $(S, r)$ as the direct limit of this system as $H$ decreases,

$$
S H^{[a, b)}(S, r):=\lim _{\longrightarrow} H F^{[a, b)}(H) .
$$

Geometrically, decreasing sequences in $A d_{r e g}(S, r)$ tend (pointwise) to $-r \chi_{B(S)}$, where $\chi_{B(S)}$ is the characteristic function of the bounded component $B(S)$.

An inclusion $S_{1} \subset B\left(S_{2}\right)$ of two hypersurfaces induces a natural inclusion $A d_{r e g}\left(S_{1}, r\right) \subset A d_{r e g}\left(S_{2}, r\right)$, and thus a homomorphism

$$
\rho\left(S_{1}, S_{2}\right): S H^{[a, b)}\left(S_{1}, r\right) \rightarrow S H^{[a, b)}\left(S_{2}, r\right) .
$$

For three hypersurfaces satisfying $S_{1} \subset B\left(S_{2}\right), S_{2} \subset B\left(S_{3}\right)$ we have

$$
\rho\left(S_{2}, S_{3}\right) \circ \rho\left(S_{1}, S_{2}\right)=\rho\left(S_{1}, S_{3}\right) .
$$

Theorem 1 will be an easy consequence of the following result about the nontriviality of certain symplectic homology groups. 


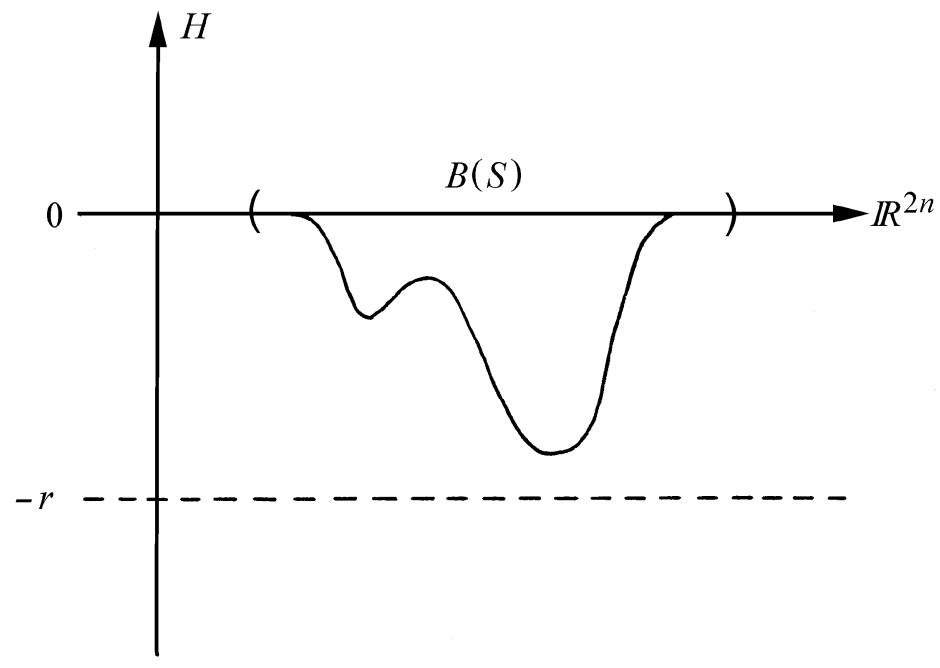

Fig. 2.

Theorem 2 (localization of symplectic homology). For $n \geq 2$ and $c \neq 0$ there exist positive numbers $a, b, \delta, r$ (which can be chosen arbitrarily small) and an embedding $f: U:=[-4 b, 4 b] \times S^{1} \times B^{2 n-2}(4 b) \hookrightarrow \mathbf{R}^{2 n}$ with the following property:

Let $S_{1}, S_{2} \subset \mathbf{R}^{2 n}$ be disjoint compact, cooriented, not necessarily connected hypersurfaces such that $S_{1} \subset B\left(S_{2}\right)$. Suppose that

$$
\begin{aligned}
& S_{1} \cap f(U)=f\left(\{-a\} \times S^{1} \times B^{2 n-2}(4 b)\right), \\
& S_{2} \cap f(U)=f\left(\{+a\} \times S^{1} \times B^{2 n-2}(4 b)\right),
\end{aligned}
$$

and the coorientations agree on these intersections. Then the closed characteristics $y_{1}=f\left(\{-a\} \times S^{1} \times\{0\}\right)$ of $S_{1}$ and $y_{2}=f\left(\{+a\} \times S^{1} \times\{0\}\right)$ of $S_{2}$ give rise to nontrivial elements

$$
0 \neq\left[y_{i}^{+}\right],\left[y_{i}^{-}\right] \in H S^{[c-\delta, c+\delta)}\left(S_{i}, r\right)
$$

in symplectic homology, and the inclusion induced homomorphism

$$
\rho\left(S_{1}, S_{2}\right): H S^{[c-\delta, c+\delta)}\left(S_{1}, r\right) \rightarrow H S^{[c-\delta, c+\delta)}\left(S_{2}, r\right)
$$

maps $\left[y_{1}^{+}\right]$onto $\left[y_{2}^{+}\right]$and $\left[y_{1}^{-}\right]$onto $\left[y_{2}^{-}\right]$.

Remark. The first part of this theorem can be interpreted as follows: The 'germ of a hypersurface' $f\left(\{a\} \times S^{1} \times B^{2 n-2}(4 b)\right)$ around the closed characteristic $y_{1}$ carries nontrivial local symplectic homology which persists 


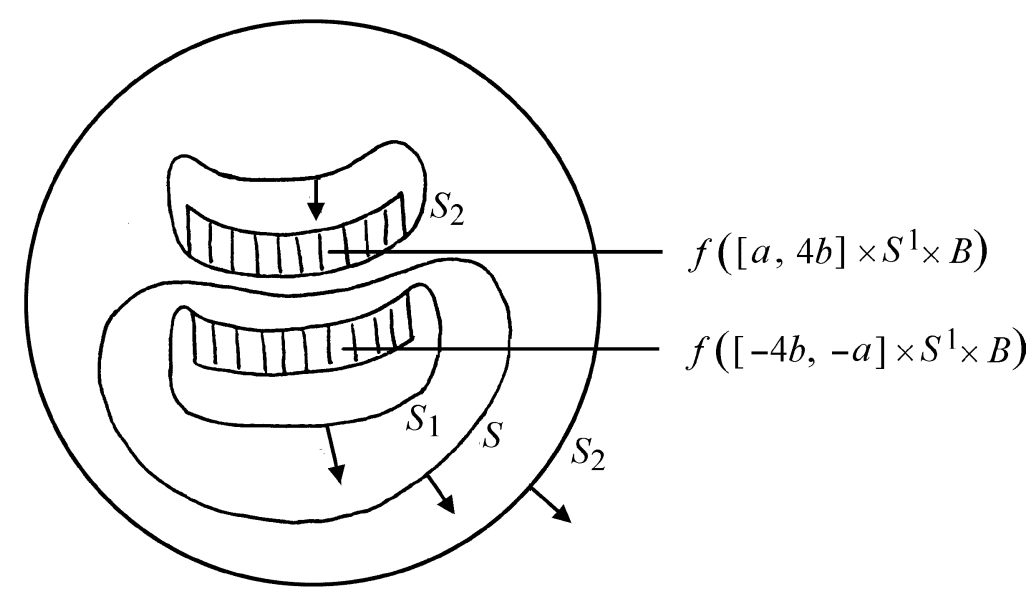

Fig. 3.

in the global symplectic homology of every closed hypersurface containing this germ.

\section{Proof of Theorem 1 assuming Theorem 2}

Let $c, a, b, \delta, r$ and $f: U \hookrightarrow \mathbf{R}^{2 n}$ be as in Theorem 2 such that $c+\delta<0$. Let $S \subset \mathbf{R}^{2 n}$ be a hypersurface as in Theorem 1. Define a hypersurface $S_{1} \subset \mathbf{R}^{2 n}$ by smoothing the corners of the boundary of $f([-4 b,-a] \times$ $\left.S^{1} \times B^{2 n-2}(4 b)\right)$. Choose the coorientation of $S_{1}$ such that it agrees with the coorientation on $f\left(\{-a\} \times S^{1} \times B^{2 n-2}(4 b)\right)$. Let the hypersurface $S_{2}$ be the disjoint union of the smoothening of the boundary of $f([a, 4 b] \times$ $\left.S^{1} \times B^{2 n-2}(4 b)\right)$ with a large sphere enclosing $S$ and $f(U)$ (see Fig. 3). Choose the coorientation of $S_{2}$ to agree with the coorientation on $f(\{a\} \times$ $\left.S^{1} \times B^{2 n-2}(4 b)\right)$ and to be outward pointing on the large sphere. Then the hypersurfaces $S_{1}$ and $S_{2}$ satisfy the hypotheses of Theorem 2, so the inclusion induced homomorphism

$$
\rho\left(S_{1}, S_{2}\right): S H^{[c-\delta, c+\delta)}\left(S_{1}, r\right) \rightarrow S H^{[c-\delta, c+\delta)}\left(S_{2}, r\right)
$$

between their symplectic homologies is nontrivial. Since

$$
\rho\left(S_{1}, S_{2}\right)=\rho\left(S, S_{2}\right) \circ \rho\left(S_{1}, S\right)
$$

this implies

$$
S H^{[c-\delta, c+\delta)}(S, r) \neq\{0\} .
$$


On the other hand, if $S$ is of restricted contact type with 1-form $\lambda$, then it can only be $\omega$-convex because

$$
\int_{S} \lambda \wedge(d \lambda)^{n-1}=\int_{B(S)} \omega^{n}>0 .
$$

In this case all closed characteristics on $S$ have positive action. Moreover, by the stability property mentioned in the introduction, $S$ possesses a tubular neighborhood $\phi([-\epsilon, \epsilon] \times S)$ such that all closed characteristics on all the hypersurfaces $S_{\rho}=\phi(\{\rho\} \times S)$ have positive action. Thus for adapted Hamiltonians which have the $S_{\rho}$ as level surfaces and are close to $-r$ respectively 0 outside the tubular neighborhood, all 1-periodic orbits have positive action. This shows that the symplectic homology groups of $S$ are trivial in all negative action intervals. In particular,

$$
H S^{[c-\delta, c+\delta)}(S, r)=\{0\},
$$

and we have a contradiction. Hence $S$ cannot be of restricted contact type.

\section{A version of the Monotonicity Lemma}

We will start with a local model for the characteristic flow near a closed characteristic. Let

$$
U:=[-4 b, 4 b] \times S^{1} \times B^{2 n-2}(4 b)
$$

be as in Theorem 2. Denote coordinates on $U$ by $(\rho, \theta, z)$ with $z=\left(z_{1}, \ldots\right.$ $\left.\ldots, z_{n-1}\right) \in \mathbf{C}^{n-1}=\mathbf{R}^{2 n-2}, z_{j}=x_{j}+i y_{j}$. We equip $U$ with the symplectic form

$$
\begin{aligned}
\omega & :=d \theta \wedge d \rho+d \theta \wedge \sum_{j=1}^{n-1} \alpha_{j}\left(x_{j} d x_{j}+y_{j} d y_{j}\right)+\omega_{2 n-2}, \\
& =d \theta \wedge d \rho+d \theta \wedge d\left(\frac{1}{2} \sum_{j=1}^{n-1} \alpha_{j}\left|z_{j}\right|^{2}\right)+\omega_{2 n-2},
\end{aligned}
$$

where $\omega_{2 n-2}=\sum_{j=1}^{n-1} d x_{j} \wedge d y_{j}$ is the standard symplectic form on $\mathbf{C}^{n}$, and

$$
\alpha_{1}, \ldots, \alpha_{n-1} \in \mathbf{R}^{+} \backslash 2 \pi \mathbf{Z}
$$

real numbers which are not multiples of $2 \pi$.

The Hamiltonian vector field of the function $\rho: U \rightarrow \mathbf{R}$ is given by

$$
X:=\frac{\partial}{\partial \theta}-\sum_{j=1}^{n-1} \alpha_{j}\left(y_{j} \frac{\partial}{\partial x_{j}}-x_{j} \frac{\partial}{\partial y_{j}}\right),
$$


as the following calculation shows:

$$
\begin{aligned}
i_{X} \omega= & d \rho+\sum_{j=1}^{n-1}\left[\left(\alpha_{j}\right)^{2}\left(x_{j} y_{j}-y_{j} x_{j}\right) d \theta+\alpha_{j}\left(x_{j} d x_{j}+y_{j} d y_{j}\right)\right. \\
& \left.-\alpha_{j}\left(y_{j} d y_{j}+x_{j} d x_{j}\right)\right] \\
= & d \rho .
\end{aligned}
$$

The components of $X$ can also be written as

$$
X=\left(0,1, i \alpha_{1} z_{1}, \ldots, i \alpha_{n-1} z_{n-1}\right) \in \mathbf{R} \times \mathbf{R} \times \mathbf{C}^{n-1} .
$$

Consider the germs of hypersurfaces

$$
U_{\rho}:=\{\rho\} \times S^{1} \times B^{2 n-2}(4 b) .
$$

If they are cooriented by the gradient of the function $\rho$, the Hamiltonian vector field $X$ is a positive (i.e. $\omega(X, \nabla \rho)>0$ ) generator of the characteristic foliations on all the hypersurfaces $U_{\rho}$. Every $U_{\rho}$ carries the closed characteristic

$$
x_{\rho}(t)=(\rho, t, 0), t \in[0,1] .
$$

The linearization at $\left(\rho, \theta_{0}, 0\right)$ of the Poincaré return map of $X$ on the transverse section $\{0\} \times\left\{\theta_{0}\right\} \times B^{2 n-2}(4 b)$ to $x_{\rho}$ in $U_{\rho}$ is the linear map of $\mathbf{C}^{n-1}$ given by the diagonal matrix

$$
\Delta:=\operatorname{diag}\left(e^{i \alpha_{1}}, \ldots, e^{i \alpha_{n-1}}\right) .
$$

Since the $\alpha_{j}$ are not multiples of $2 \pi$, the matrix $\Delta$ does not have 1 in its spectrum. So the $x_{\rho}$ are nondegenerate closed characteristics.

For a given $c \neq 0$ choose a 1 -form $\lambda_{c}$ on $U$ satisfying

$$
\begin{aligned}
d \lambda_{c} & =0 \omega, \\
-\int_{x_{0}} \lambda_{c} & =c .
\end{aligned}
$$

Define the action of a loop $x: S^{1} \rightarrow U$ by

$$
A(x):=-\int_{x} \lambda_{c} .
$$

In particular, the closed characteristic $x_{0}$ has action $c$.

The exact symplectic manifold $\left(U, d \lambda_{c}\right)$ can easily be exact symplectically embedded in $\left(\mathbf{R}^{2 n}, d \lambda_{2 n}\right)$ : Let $y_{0}: S^{1} \rightarrow \mathbf{R}^{2 n}$ be an embedded loop with $-\int_{y_{0}} \lambda_{2 n}=c$. By the symplectic neighborhood theorem (see [We1]) there exists an embedding $f: U=[-4 b, 4 b] \times S^{1} \times B^{2 n-2}(4 b) \hookrightarrow \mathbf{R}^{2 n}$ such that $f\left(x_{0}(t)\right)=y_{0}(t)$, and $f^{*} \omega_{2 n}=\omega$ with the form $\omega$ defined above. 

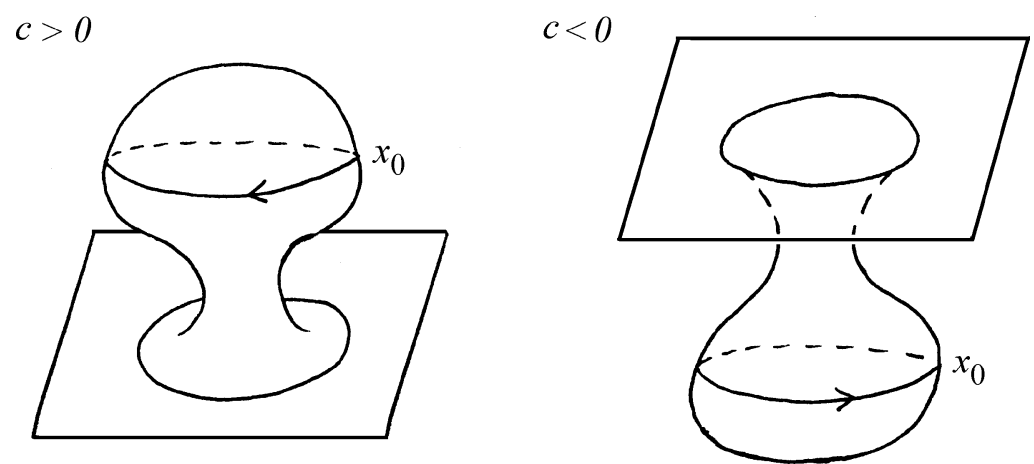

Fig. 4.

So on $U$ we have $d\left(f^{*} \lambda_{2 n}\right)=d \lambda_{c}$ and $\int_{x_{0}} f^{*} \lambda_{2 n}=\int_{x_{0}} \lambda_{c}$. Since $x_{0}$ generates the first homology of $U$, this implies that $f^{*} \lambda_{2 n}$ and $\lambda_{c}$ differ by an exact 1 -form.

For later use let us give a more explicit embedding of $U$ in $\mathbf{R}^{2 n}$.

Lemma 1. For every $c \neq 0$ and $n \geq 2$ there exists an embedding $f$ : $[-4 b, 4 b] \times \mathbf{R}^{2 n-1} \hookrightarrow \mathbf{R}^{2 n}$ and a tubular neighborhood $S^{1} \times B^{2 n-2}(4 b) \cong$ $V \subset \mathbf{R}^{2 n-1}$ of an embedded loop such that

(i) $f=\mathrm{id}$ outside some compact subset,

(ii) $f:\left(U=[-4 b, 4 b] \times V, d \lambda_{c}\right) \hookrightarrow\left(\mathbf{R}^{2 n}, d \lambda_{2 n}\right)$ is exact symplectic.

Remark. In particular, the hypersurface $f\left(\{0\} \times \mathbf{R}^{2 n-1}\right)$ contains a closed characteristic $x_{0}$ of action $c$ near which the hypersurface looks like $U_{0}$ above (see Fig. 4).

Proof. For $c>0$ consider the function

$$
\rho(z):=c-\frac{1}{2} \sum_{j=1}^{n} \alpha_{j}\left|z_{j}\right|^{2}
$$

on $\mathbf{C}^{n}$, where $\alpha_{n}=2 \pi$, and $\alpha_{1}, \ldots, \alpha_{n-1}$ are the positive numbers from above. Fix $c$, and pick $b>0$ small enough such that the level sets

$$
E_{a}:=\left\{z \in \mathbf{C}^{n} \mid \rho(z)=a\right\},
$$

$a \in[-4 b, 4 b]$, form a family of ellipsoids around the origin. Each ellipsoid $E_{a}$ carries the closed characteristic

$$
x_{a}(t)=\left(0, \ldots, 0, r_{a} e^{2 \pi i t}\right), \quad t \in[0,1],
$$

where $r_{a}$ is determined by the equation

$$
c-\frac{1}{2} r_{a}^{2}=a .
$$


The action of the characteristic $x_{0}$ is

$$
\begin{aligned}
A\left(x_{0}\right) & =-\int_{x_{0}} \lambda_{2 n} \\
& =-\frac{1}{2} \int_{0}^{1} r_{0}^{2}\left(\cos ^{2} t+\sin ^{2} t\right) d t \\
& =-\frac{1}{2} r_{0}^{2} \\
& =-c .
\end{aligned}
$$

In a tubular neighborhood $U$ of all the characteristics $x_{a}$ we can choose polar coordinates $z_{n}=r e^{2 \pi i \theta}$ in the last component. The form $\omega_{2 n}$ becomes

$$
\left.\omega_{2 n}\right|_{U}=2 \pi r d r \wedge d \theta+\omega_{2 n-2} .
$$

Now we take $\rho$ as a new independent coordinate and eliminate $r$. Pick $U$ of the form $U=[-4 b, 4 b] \times S^{1} \times B^{2 n-2}(4 b)$ in the coordinates $\left(\rho, \theta, z_{1}, \ldots\right.$ $\left.\ldots, z_{n-1}\right)$. From

$$
d \rho=-2 \pi r d r-\sum_{j=1}^{n-1} \alpha_{j}\left(x_{j} d x_{j}+y_{j} d y_{j}\right)
$$

we find the expression for $\omega_{2 n}$ in the new coordinates $\left(\rho, \theta, z_{1}, \ldots, z_{n-1}\right)$,

$$
\left.\omega_{2 n}\right|_{U}=d \theta \wedge d \rho+d \theta \wedge \sum_{j=1}^{n-1} \alpha_{j}\left(x_{j} d x_{j}+y_{j} d y_{j}\right)+\omega_{2 n-2},
$$

which is the form $\omega$ from above. This shows that the 1-form $\left.\lambda_{2 n}\right|_{U}-\lambda_{-c}$ is closed. Since $A\left(x_{0}\right)=-c$, it vanishes on the generator $x_{0}$ of $H_{1}(U)$, so it is exact.

So far we have constructed a foliated family of ellipsoids $\left(E_{a}\right)_{a \in[-4 b, 4 b]} \subset$ $\mathbf{R}^{2 n}$ containing a set $U=[-4 b, 4 b] \times S^{1} \times B^{2 n-2}(4 b)$ which satisfies (ii) of the lemma. Now translate each ellipsoid $E_{a}$ in the negative $x_{1}$-direction (by the same amount for all $a$ ) until it lies below the hypersurface $\left\{x_{1}=\right.$ a) $\subset \mathbf{R}^{2 n}$. Cut out from $E_{a}$ a small $(2 n-1)$-ball around the north pole (the point with the maximal value of $x_{1}$ ), and cut out from the hypersurface $\left\{x_{1}=a\right\}$ a small ball around $(a, 0, \ldots, 0)$. Connect the two hypersurfaces along the holes as shown in Fig. 4. Since increasing values of $a$ correspond to smaller ellipsoids, this connected sum can be performed simultaneously for all the ellipsoids $E_{a}$ as a foliated family.

This finishes the proof in the case of negative action $-c$. In the case of positive action $c$ replace the function $\rho$ by $-\rho$. The positively oriented closed characteristic $x_{0}$ then has action $+c$. Now increasing values of $a$ correspond to bigger ellipsoids. So if we translate them in the positive $x_{1}$-direction, then 
we can connect them to the hyperplanes $\left\{x_{1}=a\right\}$ as before, cutting out small balls around the south pole.

The proof of Theorem 2 depends on an estimate of the energy of pseudoholomorphic curves in $U=[-4 b, 4 b] \times S^{1} \times B^{2 n-2}(4 b)$. We will identify $U$ with its image $f(U) \subset \mathbf{R}^{2 n}$ constructed in Lemma 1. Let $H: \mathbf{R} \times$ $S^{1} \times \mathbf{R}^{2 n} \rightarrow \mathbf{R}$ be a smooth Hamiltonian satisfying (H1-2). Moreover, suppose that for $x=(\rho, \theta, z) \in U$ we have $H(s, t, x)=h(s, \rho)$, where $h: \mathbf{R} \times[-4 b, 4 b] \rightarrow \mathbf{R}$ is a smooth function with

$$
\left\{\begin{array}{c}
-\frac{|c|}{4} \leq h(s, \rho) \leq 0 \text { for all }(s, \rho) ; \\
h(s, \rho)=h^{ \pm}(s) \text { for } \pm \rho \in[2 b, 4 b] .
\end{array}\right\}
$$

Let $J$ be an $(s, t)$-dependent almost complex structure on $\mathbf{R}^{2 n}$ satisfying (J1-2), and such that for $x \in U, J(s, t, x): T_{x} U \rightarrow T_{x} U$ is given by

$$
\left\{\begin{aligned}
J(s, t, x) \cdot \frac{\partial}{\partial \rho} & =X(x), \\
J(s, t, x) \cdot X(x) & =-\frac{\partial}{\partial \rho}, \\
J(s, t, x) \cdot w & =-i w \text { for } w \in\{0\} \times\{0\} \times \mathbf{C}^{n-1} .
\end{aligned}\right\}
$$

Consider smooth maps $\hat{u}: Z=\mathbf{R} \times S^{1} \rightarrow \mathbf{R}^{2 n}$ satisfying (u1-2), where $x_{i}$ are 1-periodic solutions of $\dot{x}_{i}(t)=X_{H_{i}}\left(t, x_{i}(t)\right)$. Moreover, suppose that

$$
\begin{gathered}
x_{1}(t)=\left(\rho_{1}, t, 0\right) \in U \text { for some } \rho_{1} \in[-2 b, 2 b] ; \\
x_{2} \text { does not meet } U ; \\
A_{H}\left(x_{2}\right)-A_{H}\left(x_{1}\right) \leq \frac{|c|}{4} .
\end{gathered}
$$

Proposition 1 ('Hamiltonian Monotonicity Lemma'). There exists a constant $\kappa$ depending only on the numbers $\alpha_{1}, \ldots, \alpha_{n-1} \in \mathbf{R}^{+} \backslash 2 \pi \mathbf{Z}$ such that for all sufficiently small $b>0$ the following holds:

For all $H, J$ and $\hat{u}$ satisfying (H1-2), (H4), (J1-2), (J4) and (u1-5),

$$
\int_{Z}\left|\hat{u}_{s}\right|^{2} d s d t \geq \kappa b^{2} .
$$

The statement remains true if the roles of $x_{1}$ and $x_{2}$ are interchanged in (u3-5).

Remarks. 1. The corresponding statement for $H \equiv 0$ is the classical Monotonicity Lemma for pseudo-holomorphic curves ([Gr], inequality (14); [Hu], Chapter II, Theorem 1.3). However, this case is excluded by the hypothesis of Proposition 1 on the nonconstant periodic solution $x_{1}$. 
2. It would be interesting to know under which weaker hypotheses on the Hamiltonian flow near $x_{1}$ the conclusion of Proposition 1 remains true. It should definitely be sufficient that $x_{1}$ is nondegenerate and stable in the sense that the forward and backward orbit of any point which is $\delta$-close to $x_{1}$ remains $\epsilon$-close to $x_{1}$. In view of the classical Monotonicity Lemma it seems plausible that the nondegeneracy assumption can be removed.

Question: Does Proposition 1 remain valid without any assumption on $H$ near $x_{1}$ ?

Idea of the proof. As the proof of this proposition will occupy the remainder of this section, let us first describe the idea. By the asymptotic conditions on $\hat{u}$, there exist values of $s$ for which the loops $y(t):=\hat{u}(s, t)$ meet the 'annulus' $\{b \leq|z| \leq 2 b\} \subset U$. Once we have a uniform estimate from below,

$$
\int_{0}^{1}\left|\dot{y}-X_{H}(y)\right| d t \geq \kappa b,
$$

for the deviation of such $y$ from a periodic orbit, the energy estimate will follow by integration over $s$. To get the estimate (*), we have to consider two different cases. Either the loop $y$ stays in $U$ all the time; then the estimate follows from the the nondegeneracy assumption on $x_{1}$ which implies that the only periodic orbits in $U$ are contained in the set $\{z=0\}$. Or the loop $y$ leaves $U$; then $(*)$ follows from the stability assumption on $x_{1}$ which implies that no orbit leaves the set $U$.

Let us first focus our attention to the local hypersurface

$$
S^{1} \times B^{2 n-2}(3 b) \cong\{0\} \times S^{1} \times B^{2 n-2}(3 b) \subset U,
$$

where we have dropped the variable $\rho=0$ from the notation. For $x \in$ $S^{1} \times B^{2 n-2}(3 b)$ denote by

$$
\pi_{x}: T_{x}\left(\left(S^{1} \times B^{2 n-2}(3 b)\right) \cong \mathbf{R} \times \mathbf{C}^{n-1} \rightarrow \mathbf{C}^{n-1}\right.
$$

the projection along $X(x)$. In complex coordinates we have $x=\left(\theta, z_{1}, \ldots\right.$ $\left.\ldots, z_{n-1}\right)$, the components of $X$ are

$$
X(x)=\left(1, i \alpha_{1} z_{1}, \ldots, i \alpha_{n-1} z_{n-1}\right),
$$

and the projection $\pi$ is given explicitly by

$$
\pi_{x}\left(v, w_{1}, \ldots, w_{n-1}\right)=\left(w_{1}-i v \alpha_{1} z_{1}, \ldots, w_{n-1}-i v \alpha_{n-1} z_{n-1}\right) .
$$

Lemma 2. Let

$$
\begin{aligned}
x, y:[0, d] & \rightarrow S^{1} \times B^{2 n-2}(3 b), \\
x(t) & =(\theta(t), z(t)),
\end{aligned}
$$




$$
y(t)=(\theta(t), w(t))
$$

be two smooth curves, having the same $\theta$-component, such that y satisfies

$$
\pi_{y(t)} \dot{y}(t)=0 \text { for all } t \in[0, d] .
$$

Then

$$
\left|\frac{d}{d t}\right| z(t)-w(t)|| \leq\left|\pi_{x(t)} \dot{x}(t)\right|
$$

for every $t \in[0, d]$ with $|z(t)-w(t)|>0$. In particular,

$$
\|z-w\|_{C^{0}} \leq \int_{0}^{d}\left|\pi_{x(t)} \dot{x}(t)\right| d t+|z(0)-w(0)| .
$$

Proof. The equation $\pi_{y(t)} \dot{y}(t)=0$ is given explicitly by

$$
\dot{w}_{j}(t)-i \dot{\theta}(t) \alpha_{j} w_{j}(t)=0 \text { for } j=1, \ldots, n-1 .
$$

Using this, we can calculate

$$
\begin{aligned}
\frac{d}{d t}\left|z_{j}(t)-w_{j}(t)\right|^{2}= & 2\left\langle z_{j}(t)-w_{j}(t), \dot{z}_{j}(t)-\dot{w}_{j}(t)\right\rangle \\
= & 2\left\langle z_{j}(t)-w_{j}(t), \dot{z}_{j}(t)-i \dot{\theta}(t) \alpha_{j} z_{j}(t)\right\rangle \\
& +2\left\langle z_{j}(t)-w_{j}(t), i \dot{\theta}(t) \alpha_{j} z_{j}(t)-i \dot{\theta}(t) \alpha_{j} w_{j}(t)\right\rangle \\
= & 2\left\langle z_{j}(t)-w_{j}(t), \dot{z}_{j}(t)-i \dot{\theta}(t) \alpha_{j} z_{j}(t)\right\rangle
\end{aligned}
$$

since $\langle v, i r v\rangle=0$ for any $v \in \mathbf{C}$ and $r \in \mathbf{R}$. Summation over $j$ yields

$$
\begin{aligned}
\left|\frac{d}{d t}\right| z(t)-\left.w(t)\right|^{2} \mid & =\left|2\left\langle z(t)-w(t), \pi_{x(t)} \dot{x}(t)\right\rangle\right| \\
& \leq 2|z(t)-w(t)|\left|\pi_{x(t)} \dot{x}(t)\right|,
\end{aligned}
$$

from which the first statement follows. The second statement follows by integration over $t$.

Recall that the action of a loop $x: S^{1} \rightarrow U$ is given by $A(x)=-\int_{x} \lambda_{c}$, for a 1-form $\lambda_{c}$ on $U$ with $d \lambda_{c}=\omega$ and $-\int_{x_{0}} \lambda_{c}=c$, where $x_{0}(t)=$ $(0, t, 0), t \in[0,1]$.

Lemma 3. There exists a constant $\kappa>0$ depending only on the numbers $\alpha_{1}, \ldots, \alpha_{n-1}$ such that for all sufficiently small $b>0$ the following holds: Suppose that $x=(\theta, z):[0, d] \rightarrow S^{1} \times B^{2 n-2}(3 b)$ is a smooth curve satisfying

$$
b \leq|z(0)| \leq 2 b
$$

and one of the following two conditions:

(a) $|z(d)|=3 b$, or 
(b) $x$ is closed, and $\left|A(x)-A\left(x_{0}\right)\right| \leq \frac{3|c|}{4}$.

Then

$$
\int_{0}^{d}\left|\pi_{x(t)} \dot{x}(t)\right| d t \geq \kappa b .
$$

Proof. (a) In Case a we have $|z(d)|=3 b$ and $|z(0)| \leq 2 b$. So Lemma 2 applied to the curves $x(t)$ and $y(t)=(\theta(t), 0)$ yields

$$
3 b \leq \int_{0}^{d}\left|\pi_{x(t)} \dot{x}(t)\right| d t+2 b,
$$

thus

$$
\int_{0}^{d}\left|\pi_{x(t)} \dot{x}(t)\right| d t \geq b
$$

(b) Now suppose that $x$ is closed and satisfies (b). Arguing by contradiction, let us assume that

$$
\int_{S^{1}}\left|\pi_{x(t)} \dot{x}(t)\right| d t<\kappa b
$$

where $\kappa$ will be chosen later.

After a shift in $t$ we may assume that $\theta(0)=0$. Consider the universal covering $\mathbf{R} \rightarrow S^{1}=\mathbf{R} / \mathbf{Z}$, and let $\hat{\theta}:[0,1] \rightarrow \mathbf{R}$ be the lift of $\theta$ with $\hat{\theta}(0)=0$. Then

$$
\hat{\theta}(1)=l
$$

for some integer $l$ which is called the winding number of $\theta$.

Claim. For $b$ sufficiently small, $\theta$ has winding number $l=1$.

Proof. Define $u:[0,1] \times S^{1} \rightarrow S^{1} \times B^{2 n-2}(3 b)$,

$$
u(s, t):=(\theta(t), s z(t)),
$$

and compute the action difference

$$
\begin{aligned}
A(x)-A(u(0, \cdot))= & -\int_{[0,1] \times S^{1}} u^{*} \omega \\
= & -\int_{0}^{1} \int_{0}^{1} \omega(u)((0, z),(\dot{\theta}, s \dot{z})) d s d t \\
= & -\int_{0}^{1} \int_{0}^{1}\left[-\sum_{j=1}^{n-1} \alpha_{j} \dot{\theta}\left(s x_{j}^{2}+s y_{j}^{2}\right)\right. \\
& \left.+\omega_{2 n-2}(z, s \dot{z})\right] d s d t \\
= & \frac{1}{2} \int_{0}^{1}\left[\sum_{j=1}^{n-1} \alpha_{j} \dot{\theta}\left|z_{j}\right|^{2}-\omega_{2 n-2}(z, \dot{z})\right] d t
\end{aligned}
$$




$$
\begin{aligned}
= & \frac{1}{2} \int_{0}^{1} \sum_{j=1}^{n-1}\left[\alpha_{j} \dot{\theta}\left|z_{j}\right|^{2}-d x_{j} \wedge d y_{j}\left(z_{j}, \dot{z}_{j}-i \alpha_{j} \dot{\theta} z_{j}\right)\right. \\
& \left.-d x_{j} \wedge d y_{j}\left(z_{j}, i \alpha_{j} \dot{\theta} z_{j}\right)\right] d t \\
= & -\frac{1}{2} \int_{0}^{1} \sum_{j=1}^{n-1} d x_{j} \wedge d y_{j}\left(z_{j}, \dot{z}_{j}-i \alpha_{j} \dot{\theta} z_{j}\right) d t \\
= & -\frac{1}{2} \int_{0}^{1} \omega_{2 n-2}\left(z, \pi_{x} \dot{x}\right) d t .
\end{aligned}
$$

Using assumption $(* *)$ we get

$$
\begin{aligned}
|A(x)-A(u(0, \cdot))| & \leq \frac{1}{2} \int_{0}^{1}|z|\left|\pi_{x} \dot{x}\right| d t \\
& <\frac{1}{2} \cdot 3 b \cdot \kappa b \\
& <\frac{|c|}{4}
\end{aligned}
$$

for $b$ sufficiently small. On the other hand,

$$
A(u(0, \cdot))=l A\left(x_{0}\right)=l c,
$$

and therefore

$$
\begin{aligned}
\frac{|c|}{4} & >|A(x)-l c| \\
& \geq|l-1||c|-|A(x)-c| \\
& \geq|l-1||c|-\frac{3|c|}{4}
\end{aligned}
$$

by hypothesis (b). Dividing by $|c|>0$ yields $|l-1|<1$, thus $l=1$, and the claim is proved.

Now let $y:[0,1] \rightarrow S^{1} \times B^{2 n-2}(3 b)$ be the curve satisfying

$$
\left\{\begin{array}{l}
y(t)=(\theta(t), w(t)), \\
\pi_{y(t)} \dot{y}(t)=0, \\
y(0)=x(0) .
\end{array}\right\}
$$

The hypothesis that $\alpha_{1}, \ldots, \alpha_{n-1}$ are not mupliples of $2 \pi$ implies that $S^{1} \times$ $\{0\}$ is the only closed characteristic in $S^{1} \times B^{2 n-2}(3 b)$ of winding number 1. Consequently, since $\theta$ has winding number 1 and $|w(0)| \geq b$,

$$
|w(1)-w(0)| \geq \kappa b
$$


for some constant $\kappa$ depending only on $\alpha_{1}, \ldots, \alpha_{n-1}$. By the choice of $y$ we have $z(0)=w(0)$, so from assumption $(* *)$ and Lemma 2 we get

$$
\begin{aligned}
|z(1)-z(0)| & \geq|w(1)-w(0)|-|z(1)-w(1)| \\
& >\kappa b-\kappa b=0 .
\end{aligned}
$$

But this contradicts the hypothesis that $x$ is a closed curve, and Lemma 3 is proved.

Now let $H, J$, and $\hat{u}$ satisfy the hypotheses (H1-2), (H4), (J1-2), (J4) and (u1-5). Let $\Omega \subset Z$ be the set of all $z$ for which $\hat{u}(z)$ lies in the region

$$
([-4 b,-2 b] \cup[2 b, 4 b]) \times S^{1} \times B^{2 n-2}(4 b) .
$$

By (H4) the gradient of $H$ vanishes in this region. So the restriction $\left.\hat{u}\right|_{\Omega}$ is $J$-holomorphic, and its area equals

$$
\int_{\Omega}\left|\hat{u}_{s}\right|^{2} d s d t
$$

By the Monotonicity Lemma (see [Hu], Chapter II, Theorem 1.3) there exists a constant $D$ depending only on $\alpha_{1}, \ldots, \alpha_{n-1}$ such that if $\left.\hat{u}\right|_{\Omega}$ meets the set

$$
A:=\{ \pm 3 b\} \times S^{1} \times B^{2 n-2}(3 b),
$$

then its area is at least

$$
\int_{\Omega}\left|\hat{u}_{s}\right|^{2} d s d t \geq D b^{2}
$$

and Proposition 1 follows. So let us suppose from now on that $\hat{u}$ does not meet the set $A$.

Define a continuous function $d: \mathbf{R}^{2 n} \backslash A \rightarrow[0,3 b]$ by

$$
d(x):= \begin{cases}|z| & \text { for } x=(\rho, \theta, z) \in(-3 b, 3 b) \times S^{1} \times B^{2 n-2}(3 b) ; \\ 3 b & \text { otherwise }\end{cases}
$$

(see Fig. 5). Given $\hat{u}: Z \rightarrow \mathbf{R}^{2 n}$ as above, define $B: \mathbf{R} \rightarrow[0,3 b]$,

$$
B(s):=\left(\int_{0}^{1} d(\hat{u}(s, t))^{2} d t\right)^{\frac{1}{2}} .
$$

By the asymptotic conditions (u2), (u3) and (u4) we have

$$
\begin{aligned}
B(s) \rightarrow 0 & \text { as } s \rightarrow-\infty, \\
B(s) \rightarrow 3 b & \text { as } s \rightarrow+\infty .
\end{aligned}
$$




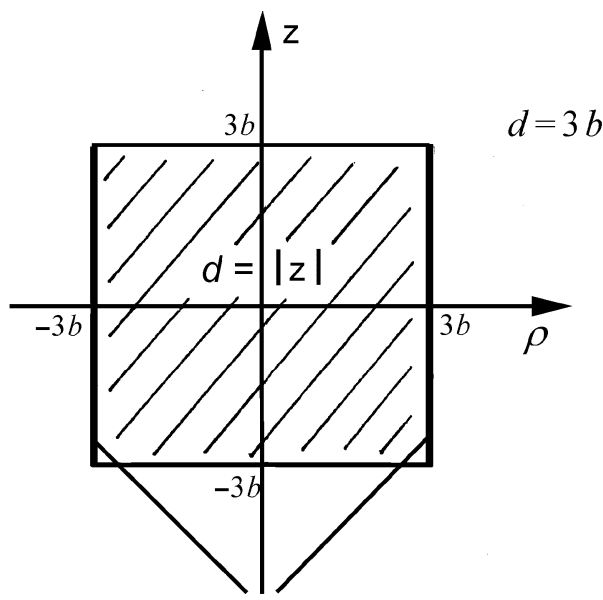

A

Fig. 5.

Thus there exist values $s_{1}<s_{2}$ such that

$$
\begin{gathered}
B\left(s_{1}\right)=b, \\
B\left(s_{2}\right)=2 b, \text { and } \\
b \leq B(s) \leq 2 b \text { for all } s \in\left[s_{1}, s_{2}\right] .
\end{gathered}
$$

Lemma 4. For each $t \in S^{1}$,

$$
d\left(\hat{u}\left(s_{2}, t\right)\right)-d\left(\hat{u}\left(s_{1}, t\right)\right) \leq \int_{s_{1}}^{s_{2}}\left|\hat{u}_{s}(s, t)\right| d s .
$$

Proof. Consider the smaller region

$$
U^{\prime}:=[-3 b, 3 b] \times S^{1} \times B^{2 n-2}(3 b) \subset U .
$$

We will distinguish several cases, for $t \in S^{1}$ fixed.

Case 1: $\hat{u}(s, t) \in U^{\prime}$ for all $s \in\left[s_{1}, s_{2}\right]$.

On $U^{\prime}$, the metric $\langle\cdot, \cdot\rangle=\omega(J \cdot, \cdot)$ is given by

$$
\begin{aligned}
& \left\langle u \frac{\partial}{\partial \rho}+v X+w, u^{\prime} \frac{\partial}{\partial \rho}+v^{\prime} X+w^{\prime}\right\rangle \\
& =\omega\left(-v \frac{\partial}{\partial \rho}+u X-i w, u^{\prime} \frac{\partial}{\partial \rho}+v^{\prime} X+w^{\prime}\right) \\
& =v v^{\prime}+u u^{\prime}+\left\langle w, w^{\prime}\right\rangle_{2 n-2},
\end{aligned}
$$


where $u, v \in \mathbf{R}, w \in\{0\} \times\{0\} \times \mathbf{C}^{n-1}$, and $\langle\cdot, \cdot\rangle_{2 n-2}$ is the Euclidean metric on $\mathbf{C}^{n-1}$.

Writing $\hat{u}$ in $U^{\prime}$ as

$$
\hat{u}=(\rho, u)=(\rho, \theta, z),
$$

we obtain

$$
\begin{aligned}
\hat{u}_{s} & =\rho_{s} \frac{\partial}{\partial \rho}+u_{s} \\
& =\rho_{s} \frac{\partial}{\partial \rho}+\theta_{s} X(u)+\pi_{u} u_{s},
\end{aligned}
$$

and therefore

$$
\begin{aligned}
\left|\hat{u}_{s}\right|^{2} & =\left|\rho_{s}\right|^{2}+\left|\theta_{s}\right|^{2}+\left|\pi u_{s}\right|^{2} \\
& \geq\left|\pi u_{s}\right|^{2} .
\end{aligned}
$$

Using Lemma 2 we infer

$$
\begin{aligned}
d\left(\hat{u}\left(s_{2}, t\right)\right)-d\left(\hat{u}\left(s_{1}, t\right)\right) & =\left|z\left(s_{2}, t\right)\right|-\left|z\left(s_{1}, t\right)\right| \\
& \leq \int_{s_{1}}^{s_{2}}\left|\frac{\partial}{\partial s}\right| z(s, t)|| d s \\
& \leq \int_{s_{1}}^{s_{2}}\left|\pi u_{s}(s, t)\right| d s \\
& \leq \int_{s_{1}}^{s_{2}}\left|\hat{u}_{s}(s, t)\right| d s .
\end{aligned}
$$

Case 2: $\hat{u}\left(s_{1}, t\right), \hat{u}\left(s_{2}, t\right) \in U^{\prime}$, but $\hat{u}(s, t) \notin U^{\prime}$ for some $s \in\left[s_{1}, s_{2}\right]$.

Since $\hat{u}$ avoids the set $A$, it must leave and enter $U^{\prime}$ through

$$
B:=[-3 b, 3 b] \times S^{1} \times \partial B^{2 n-2}(3 b) .
$$

Choose $s_{1} \leq s_{1}^{\prime} \leq s_{2}^{\prime} \leq s_{2}$ such that

$$
\begin{gathered}
\hat{u}\left(s_{1}^{\prime}, t\right), \hat{u}\left(s_{2}^{\prime}, t\right) \in B, \\
\hat{u}(s, t) \in U^{\prime} \text { for all } s \in\left[s_{1}, s_{1}^{\prime}\right] \cup\left[s_{2}^{\prime}, s_{2}\right] .
\end{gathered}
$$

Then by Case 1

$$
\begin{aligned}
& d\left(\hat{u}\left(s_{2}, t\right)\right)-d\left(\hat{u}\left(s_{1}, t\right)\right) \\
& =d\left(\hat{u}\left(s_{2}, t\right)\right)-d\left(\hat{u}\left(s_{2}^{\prime}, t\right)\right)+d\left(\hat{u}\left(s_{1}^{\prime}, t\right)\right)-d\left(\hat{u}\left(s_{1}, t\right)\right) \\
& \leq \int_{s_{1}}^{s_{1}^{\prime}}\left|\hat{u}_{s}(s, t)\right| d s+\int_{s_{2}^{\prime}}^{s_{2}}\left|\hat{u}_{s}(s, t)\right| d s \\
& \leq \int_{s_{1}}^{s_{2}}\left|\hat{u}_{s}(s, t)\right| d s .
\end{aligned}
$$


Case 3: $\hat{u}\left(s_{1}, t\right) \in U^{\prime}$, and $\hat{u}\left(s_{2}, t\right) \notin U^{\prime}$.

Choose a number $s_{1} \leq s_{1}^{\prime} \leq s_{2}$ such that

$$
\begin{gathered}
\hat{u}\left(s_{1}^{\prime}, t\right) \in B, \\
\hat{u}(s, t) \in U^{\prime} \text { for all } s \in\left[s_{1}, s_{1}^{\prime}\right] .
\end{gathered}
$$

Then

$$
\begin{aligned}
d\left(\hat{u}\left(s_{2}, t\right)\right)-d\left(\hat{u}\left(s_{1}, t\right)\right) & =d\left(\hat{u}\left(s_{1}^{\prime}, t\right)\right)-d\left(\hat{u}\left(s_{1}, t\right)\right) \\
& \leq \int_{s_{1}}^{s_{2}}\left|\hat{u}_{s}(s, t)\right| d s .
\end{aligned}
$$

Case 4: If $\hat{u}\left(s_{1}, t\right), \hat{u}\left(s_{2}, t\right) \notin U^{\prime}$, then $d\left(\hat{u}\left(s_{2}, t\right)\right)-d\left(\hat{u}\left(s_{1}, t\right)\right)=0$.

Up to interchanging the roles of $s_{1}$ and $s_{2}$, these are all the cases, and the lemma is proved.

\section{Proof of Proposition 1}

The differential equation (u1) for $\hat{u}$ yields

$$
\begin{aligned}
\frac{d}{d s} A_{H}(\hat{u}(s, \cdot)) & =\int_{S^{1}}\left|\hat{u}_{s}(s, t)\right|^{2} d t-\int_{S^{1}} H_{s}(s, t, \hat{u}) d t \\
& \geq 0
\end{aligned}
$$

by (H1). So the hypothesis (u5) implies

$$
\left|A_{H}(\hat{u}(s, \cdot))-A_{H}\left(x_{1}\right)\right| \leq \frac{|c|}{4}
$$

for all $s \in \mathbf{R}$.

Suppose that for some $s \in \mathbf{R}$ we have $\hat{u}(s, t) \in U$ for all $t \in S^{1}$. Let $u_{0}(s, t):=(0, u(s, t))$, where $\hat{u}(s, t)=(\rho(s, t), u(s, t))$. Recall that $x_{0}(t)=(0, t, 0) \in U$. With these notations,

$$
\begin{aligned}
\left|A\left(u_{0}(s)\right)-A\left(x_{0}\right)\right| \leq & \left|A(\hat{u}(s))-A\left(x_{1}\right)\right|+\left|A(\hat{u}(s))-A\left(u_{0}(s)\right)\right| \\
& +\left|A\left(x_{1}\right)-A\left(x_{0}\right)\right| \\
\leq & \left|A_{H}(\hat{u}(s))-A_{H}\left(x_{1}\right)\right| \\
& +\int_{0}^{1}\left|H(s, t, \hat{u}(s, t))-H_{1}\left(t, x_{1}(t)\right)\right| d t \\
& +3 b+3 b \\
\leq & \frac{|c|}{4}+\frac{|c|}{4}+3 b+3 b \\
\leq & \frac{3|c|}{4}
\end{aligned}
$$


for $b$ sufficiently small, where we have used the estimate above and (H4).

By the choice of $s_{1}$ and $s_{2}$ preceding Lemma 4, for each $s \in\left[s_{1}, s_{2}\right]$ there exists a $t(s) \in S^{1}$ such that

$$
b \leq d(\hat{u}(s, t(s))) \leq 2 b,
$$

i.e. $\hat{u}(s, t(s))=(\rho, \theta, z) \in U^{\prime}$ and $b \leq|z| \leq 2 b$.

If moreover $\hat{u}(s, t) \in U^{\prime}$ for all $t \in S^{1}$, then by the computation above its projection $u_{0}(s, \cdot)$ satisfies hypothesis (b) of Lemma 3.

If the curve $\hat{u}(s, \cdot)$ leaves $U^{\prime}$, then since it avoids the set $A$ it must pass through $B=[-3 b, 3 b] \times S^{1} \times \partial B^{2 n-2}(3 b)$. So in this case $\hat{u}(s, \cdot)$ satisfies (a) of Lemma 3 .

Hence by Lemma 3 for each $s \in\left[s_{1}, s_{2}\right]$

$$
\int_{I(s)}\left|\pi u_{t}\right| d t \geq \kappa b
$$

where $I(s):=\left\{t \in S^{1} \mid \hat{u}(s, t) \in U^{\prime}\right\}$. On the other hand, on $U^{\prime}$ the Hamiltonian vector field of $H$ is given by

$$
\begin{aligned}
X_{H}(\rho, x) & =J(\rho, x) \nabla_{J} H(s, \rho, x) \\
& =J(\rho, x) h_{\rho}(s, \rho) \frac{\partial}{\partial \rho} \\
& =h_{\rho}(s, \rho) X(x) .
\end{aligned}
$$

Hence for $t \in I(s)$,

$$
\begin{aligned}
\left|\hat{u}_{t}-X_{H}(\hat{u})\right|^{2} & =\left|\rho_{t}\right|^{2}+\left|\theta_{t}-h_{\rho}(s, \rho)\right|^{2}+\left|\pi u_{t}\right|^{2} \\
& \geq\left|\pi u_{t}\right|^{2} .
\end{aligned}
$$

Integrating over $t$ we obtain

$$
\left\|\hat{u}_{t}(s, \cdot)-X_{H}(\hat{u}(s, \cdot))\right\|_{L^{1}(d t)} \geq \kappa b
$$

for all $s \in\left[s_{1}, s_{2}\right]$. So we can estimate

$$
\begin{aligned}
& \int_{Z}\left|\hat{u}_{s}(s, t)\right|^{2} d s d t \\
& \geq \int_{s_{1}}^{s_{2}} \int_{0}^{1}\left|\hat{u}_{s}(s, t)\right|^{2} d s d t \\
& =\int_{s_{1}}^{s_{2}}\left\|\hat{u}_{s}(s, \cdot)\right\|_{L^{2}(d t)}\left\|\hat{u}_{t}(s, \cdot)-X_{H}(\hat{u}(s, \cdot))\right\|_{L^{2}(d t)} d s \\
& \geq \int_{s_{1}}^{s_{2}}\left\|\hat{u}_{s}(s, \cdot)\right\|_{L^{2}(d t)}\left\|\hat{u}_{t}(s, \cdot)-X_{H}(\hat{u}(s, \cdot))\right\|_{L^{1}(d t)} d s
\end{aligned}
$$




$$
\begin{aligned}
& \geq \kappa b \int_{s_{1}}^{s_{2}}\left\|\hat{u}_{s}(s, \cdot)\right\|_{L^{2}(d t)} d s \\
& \geq \kappa b\left\|\int_{s_{1}}^{s_{2}}\left|\hat{u}_{s}(s, \cdot)\right| d s\right\|_{L^{2}(d t)} \\
& \geq \kappa b\left\|d\left(\hat{u}\left(s_{2}, \cdot\right)\right)-d\left(\hat{u}\left(s_{1}, \cdot\right)\right)\right\|_{L^{2}(d t)} \\
& \geq \kappa b\left(\left\|d\left(\hat{u}\left(s_{2}, \cdot\right)\right)\right\|_{L^{2}(d t)}-\left\|d\left(\hat{u}\left(s_{1}, \cdot\right)\right)\right\|_{L^{2}(d t)}\right) \\
& =\kappa b\left(B\left(s_{2}\right)-B\left(s_{1}\right)\right) \\
& =\kappa b^{2} .
\end{aligned}
$$

If the roles of $x_{1}$ and $x_{2}$ are interchanged we obtain the same estimate, and Proposition 1 is proved.

\section{Proof of Theorem 2 and Corollaries 1-4}

Let $c \neq 0$ be given. Consider Hamiltonians $H$ which satisfy (H1-4) of Sect. 3, as well as almost complex structures $J$ satisfying (J1-4).

Fix positive numbers $a \leq b$ and $r \leq \frac{|c|}{4}$. By hypothesis (H4) the Hamiltonian is given on the set $U=[-4 b, 4 b] \times S^{1} \times B^{2 n-2}(4 b)$ by $H(s, t, x)=h(s, \rho), x=(\rho, \theta, z) \in U$. To compute symplectic homology we will choose $h: \mathbf{R} \times[-4 b, 4 b] \rightarrow \mathbf{R}$ of a particular form which is similar to the Hamiltonians in [CFHW]: For every $s \in \mathbf{R}$ there exist numbers $-2 a \leq \rho_{1}<\rho_{2}<\rho_{3}<\rho_{4} \leq 2 a$ such that $k=h(s, \cdot)$ satisfies

$$
\left\{\begin{array}{l}
k \equiv \text { const }>-r \text { in }\left[-4 b, \rho_{1}\right] \\
0<k^{\prime}<1 \text { in }\left(\rho_{1}, \rho_{2}\right) \cup\left(\rho_{3}, \rho_{4}\right) \\
k^{\prime}\left(\rho_{2}\right)=1, k^{\prime \prime}\left(\rho_{2}\right)>0, k\left(\rho_{2}\right)<-r+a ; \\
k^{\prime}\left(\rho_{3}\right)=1, k^{\prime \prime}\left(\rho_{3}\right)<0, k\left(\rho_{3}\right)>-a ; \\
k^{\prime}>1 \text { in }\left(\rho_{2}, \rho_{3}\right) \\
k \equiv 0 \text { in }\left[\rho_{4}, 4 b\right]
\end{array}\right\}
$$

(see Fig. 6).

Fix a number $0<\delta<|c|$ which will be specified later, and consider Floer homology in the action interval $[c-\delta, c+\delta)$. Notice that an $s$-independent Hamiltonian satisfying (H5) possesses two particular degenerate 1-periodic orbits in $U$,

$$
\begin{aligned}
& x_{H}(t):=\left(\rho_{3}, t, 0\right) \text { and } \\
& \tilde{x}_{H}(t):=\left(\rho_{2}, t, 0\right) .
\end{aligned}
$$




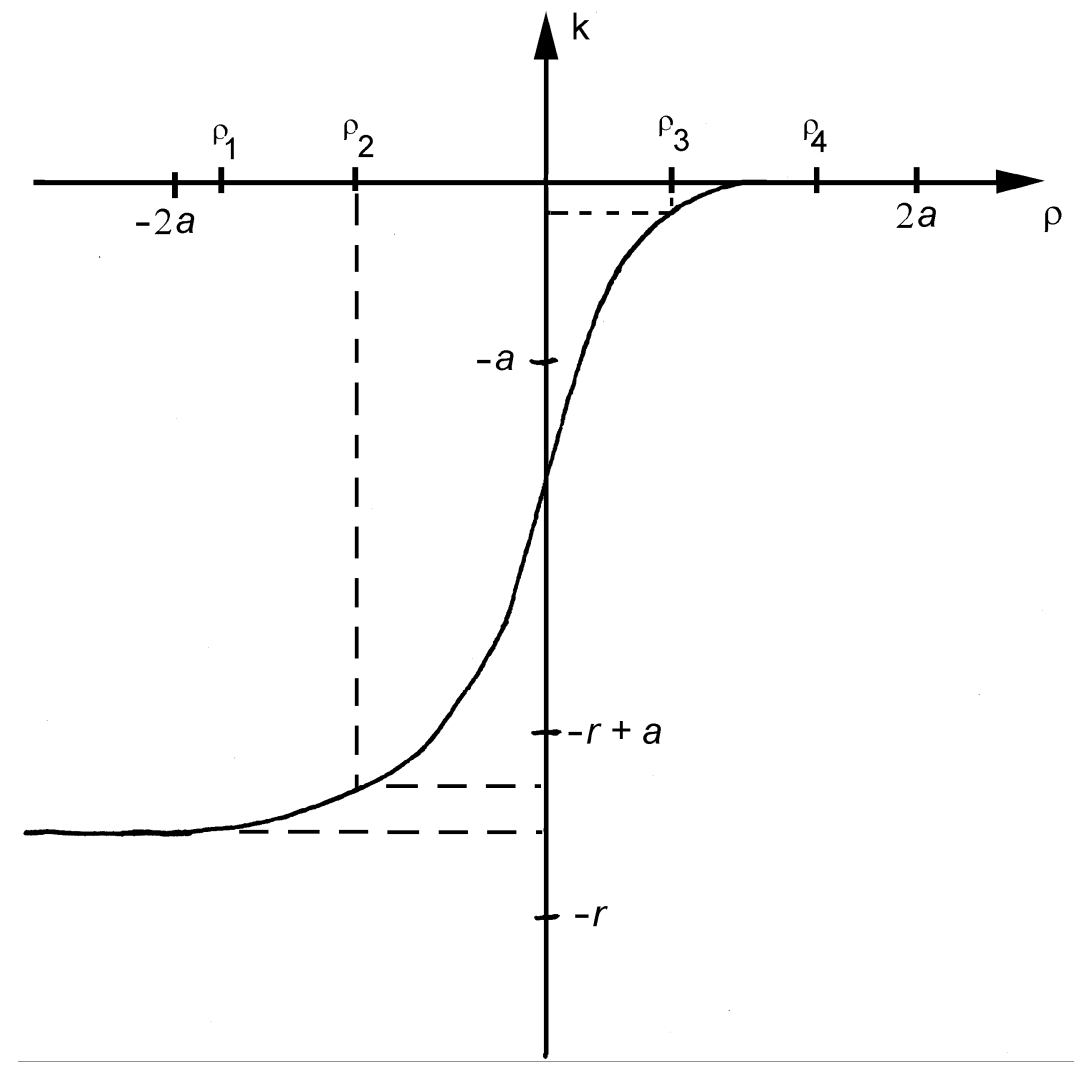

Fig. 6.

The Hamiltonian action of $x_{H}$ is

$$
\begin{aligned}
A_{H}\left(x_{H}\right) & =-\int_{x_{H}} \lambda_{c}-\int_{0}^{1} H\left(t, x_{H}\right) d t \\
& =c-\rho_{3}-h\left(\rho_{3}\right) .
\end{aligned}
$$

By (H5) this implies $c-2 a \leq A_{I I}\left(x_{I I}\right) \leq c+3 a$, hence

$$
A_{H}\left(x_{H}\right) \in(c-\delta, c+\delta)
$$

if $3 a<\delta$. As $x_{H}$ is degenerate, an $s$-independent Hamiltonian $H$ satisfying (H5) cannot be regular with respect to the action interval $[c-\delta, c+\delta)$. However, $H$ can be made regular by a $C^{\infty}$-small perturbation. We denote by

$$
H F^{[c-\delta, c+\delta)}\left(H_{r e g}\right)
$$

the Floer homology of a sufficiently small regular perturbation of $H$. Although the Floer homology may depend on the perturbation, the properties stated in the following proposition are independent of the perturbation. 
Proposition 2. Suppose that $0<4 a \leq \delta \leq \frac{r}{2} \leq \frac{|c|}{8}$ and $\delta \leq \frac{1}{2} \kappa b^{2}$, where $\kappa$ is the constant of Proposition 1. Then for every sufficiently small regular perturbation $H_{\text {reg }}$ of an s-independent Hamiltonian H satisfying (H1-5), the 1-periodic orbit $x_{H}$ yields two nontrivial elements

$$
0 \neq\left[x_{H}^{+}\right],\left[x_{H}^{-}\right] \in H F^{[c-\delta, c+\delta)}\left(H_{r e g}\right) .
$$

Moreover, for two Hamiltonians $H_{1} \geq H_{2}$ satisfying (H1-5), the induced homomorphism

$$
\begin{aligned}
\sigma\left(\left(H_{1}\right)_{r e g},\left(H_{2}\right)_{r e g}\right): & H F^{[c-\delta, c+\delta)}\left(\left(H_{1}\right)_{r e g}\right) \\
& \rightarrow H F^{[c-\delta, c+\delta)}\left(\left(H_{2}\right)_{r e g}\right)
\end{aligned}
$$

maps $\left[x_{H_{1}}^{+}\right]$onto $\left[x_{H_{2}}^{+}\right]$and $\left[x_{H_{1}}^{-}\right]$onto $\left[x_{H_{2}}^{-}\right]$.

Proof. 1. Let $H$ be an $s$-independent Hamiltonian satisfying (H1-5). Let us first determine all 1-periodic orbits of $H$ in $U$ whose actions lie in the interval $[c-\delta, c+\delta]$.

It has been shown above that the orbit $x_{H}(t)=\left(\rho_{3}, t, 0\right)$ has action $A_{H}\left(x_{H}\right) \in$ $(c-\delta, c+\delta)$ for $4 a \leq \delta$.

The action of the orbit $\tilde{x}_{H}(t)=\left(\rho_{2}, t, 0\right)$ is

$$
\begin{aligned}
A_{H}\left(\tilde{x}_{H}\right) & =c-\rho_{2}-h\left(\rho_{2}\right) \\
& \geq c+r-3 a \\
& >c+\delta
\end{aligned}
$$

for $4 a \leq \delta \leq \frac{r}{2}$.

Multiply covered orbits $x(t)=(\rho, k t, z(t)), k \geq 2$, have action

$$
\begin{aligned}
A_{H}(x) & \geq k(c-2 a) \\
& >c+\delta
\end{aligned}
$$

if $c>0$, and

$$
\begin{aligned}
A_{H}(x) & \leq k(c+2 a)+r \\
& <c-\delta
\end{aligned}
$$

if $c<0$.

The constant orbits $(r, \theta, z)$ with $\rho \in\left[\rho_{4}, 4 b\right]$ have action $-h(\rho)=0 \notin$ $[c-\delta, c+\delta]$, and the constant orbits with $\rho \in\left[-4 b, \rho_{1}\right]$ have action $-h(\rho) \approx$ $r \notin[c-\delta, c+\delta]$.

So $x_{H}$ is the only 1-periodic orbit in $U$ with action in the interval $[c-\delta, c+\delta]$. 
2. Choose an $s$-independent almost complex structure $J$ satisfying (J1-4). Suppose that $y$ is a 1-periodic orbit outside $U$, and there exists a "connecting orbit' $\hat{u}$ satisfying (u1) and

$$
\hat{u}(s, \cdot) \longrightarrow \begin{cases}x_{H} & \text { as } s \rightarrow-\infty \\ y & \text { as } s \rightarrow+\infty\end{cases}
$$

By Proposition 1, the action of $y$ is at least

$$
\begin{aligned}
A_{H}(y) & \geq A_{H}\left(x_{H}\right)+\int_{Z}\left|\hat{u}_{s}\right|^{2} d s d t \\
& >c-\delta+\kappa b^{2} \\
& \geq c+\delta
\end{aligned}
$$

because $\delta \leq \frac{1}{2} \kappa b^{2}$. Similarly, if $\hat{u}$ is a 'connecting orbit' with

$$
\hat{u}(s, \cdot) \longrightarrow \begin{cases}y & \text { as } s \rightarrow-\infty \\ x_{H} & \text { as } s \rightarrow+\infty\end{cases}
$$

then $A_{H}(y)<c-\delta$.

So there exists no 'connecting orbit' $\hat{u}$ between $x_{H}$ and any other 1-periodic solution $y \neq x_{H}$ with action in $[c-\delta, c+\delta]$. By a compactness argument, this property persists under a small perturbation of $(H, J)$ to a regular pair $(H, J)_{\text {reg. }}$. Hence the contribution of $x_{H}$ to

$$
H F^{[c-\delta, c+\delta)}\left(H_{r e g}\right)=H F^{[c-\delta, c+\delta)}\left((H, J)_{r e g}\right)
$$

equals the local Floer homology of $x_{H}$, which was shown in [CFHW] to have two generators $\left[x_{H}^{ \pm}\right]$of Conley-Zehnder indices ind $\left(\left[x_{H}^{+}\right]\right)=\operatorname{ind}\left(\left[x_{H}^{-}\right]\right)+1$. 3. Two $s$-independent pairs $\left(H_{1}, J_{1}\right),\left(H_{2}, J_{2}\right)$ satisfying (H1-5) and (J1-4) with $H_{1} \geq H_{2}$ can be connected by a monotone homotopy $(H, J)$ also satisfying (H1-5) and (J1-4). Arguing as in 2., we conclude from Proposition 1 that there exist no solutions $\hat{u}$ of (u1) connecting one of the orbits $x_{H_{1}}^{ \pm}, x_{H_{2}}^{ \pm}$ to any orbit different from them with action in $[c-\delta, c+\delta]$. In particular, the image of $\left[x_{H_{1}}^{ \pm}\right]$under the homomorphism

$$
\sigma\left(\left(H_{1}\right)_{r e g},\left(H_{2}\right)_{r e g}\right)=\sigma\left((H, J)_{r e g}\right)
$$

can be computed on the set $U$.

But during the whole homotopy the only 1-periodic orbits of $H(s, \cdot)$ in $U$ with action in $[c-\delta, c+\delta]$ are $x_{H(s,)}^{ \pm}$, and their actions remain in the open interval $(c-\delta, c+\delta)$. So $\left.\sigma\left((H, J)_{\text {reg }}\right)\right|_{U}$ is a composition of small distance isomorphisms in the sense of [FHW], and therefore an isomorphism. In view of the Conley-Zehnder indices of the orbits $x_{I_{i}}^{ \pm}$this is only possible if $\sigma\left((H, J)_{\text {reg }}\right)$ maps $\left[x_{H_{1}}^{+}\right]$onto $\left[x_{H_{2}}^{+}\right]$and $\left[x_{H_{1}}^{-}\right]$onto $\left[x_{H_{2}}^{-}\right]$. 


\section{Proof of Theorem 2}

For the given number $c \neq 0$, let $a, b, \delta, r$ and $U \subset \mathbf{R}^{2 n}$ be as in Proposition 2. Suppose that $S_{i} \subset \mathbf{R}^{2 n}$ are disjoint compact cooriented hypersurfaces intersecting $U$ as in Theorem 2 . We will compute the symplectic homology of $\left(S_{i}, r\right)$ using the cofinal system consisting of regular adapted Hamiltonians $H_{\text {reg }} \in A d_{\text {reg }}\left(S_{i}, r\right)$, where $H$ satisfies (H1-5). By Proposition 2, the Floer homology of every such Hamiltonian contains two nontrivial elements

$$
0 \neq\left[x_{H}^{ \pm}\right] \in H F^{[c-\delta, c+\delta)}\left(H_{r e g}\right)
$$

corresponding to the closed characteristic $y_{i}$ of Theorem 2. Moreover for two Hamiltonians $H_{1} \geq H_{2}$ satisfying (H1-5),

$$
\sigma\left(\left(H_{1}\right)_{r e g},\left(H_{2}\right)_{r e g}\right) \cdot\left[x_{H_{1}}^{ \pm}\right]=\left[x_{H_{2}}^{ \pm}\right] .
$$

It follows from the definition of the direct limit that the $\left[x_{H}^{ \pm}\right]$yield nontrivial elements

$$
0 \neq\left[y_{i}^{ \pm}\right] \in S H^{[c-\delta, c+\delta)}\left(S_{i}, r\right) .
$$

If $H_{\text {reg }} \in A d_{\text {reg }}\left(S_{1}, r\right)$, where $H$ satisfies (H1-5), then (again by Proposition 2) the elements $\left[x_{H}^{ \pm}\right]$also persist under savethe direct limit over $A d_{\text {reg }}\left(S_{2}, r\right)$, giving rise to the elements $\left[y_{2}^{ \pm}\right]$. This proves that the inclusion induced homomorphism

$$
\rho\left(S_{1}, S_{2}\right): S H^{[c-\delta, c+\delta)}\left(S_{1}, r\right) \rightarrow S H^{[c-\delta, c+\delta)}\left(S_{2}, r\right)
$$

maps $\left[y_{1}^{ \pm}\right]$onto $\left[y_{2}^{ \pm}\right]$.

To prove Corollary 1, we need some elementary properties of the Hausdorff metric $d_{H}$ formulated in the following two lemmas.

Given a connected topological space $X$ and two disjoint subsets $A_{1}, A_{2}$ of $X$, we say that a subset $B$ of $X$ separates $A_{1}$ from $A_{2}$ if $X \backslash B$ has precisely 2 connected components $U_{1}, U_{2}$ with $U_{i}$ containing $A_{i}$ for $i=1,2$.

Lemma 5. (a) Let $(X, d)$ be a bounded connected metric space, and equip $[0,1] \times X$ with the metric $d((r, x),(s, y)):=|r-s|+d(x, y)$. Then for any closed subset $A$ of $[0,1] \times X$ which separates $\{0\} \times X$ from $\{1\} \times X$,

$$
d_{H}(A,\{1\} \times X)=\sup _{a \in A} d(a,\{1\} \times X) .
$$

Proof. Let $b=(1, x) \in\{1\} \times X$. If the line $[0,1] \times\{x\}$ did not intersect $A$ then it would connect $\{0\} \times X$ with $\{1\} \times X$ in $([0,1] \times X) \backslash A$, in contradiction to the separation property of $A$. Thus there exists a number $r_{0} \in[0,1]$ such that

$$
a_{0}=\left(r_{0}, x\right) \in A \text {. }
$$


We obtain

$$
\begin{aligned}
d(b, A) & \leq d\left(b, a_{0}\right) \\
& =\left|1-r_{0}\right| \\
& =d\left(a_{0},\{1\} \times X\right) \\
& \leq \sup _{a \in A} d(a,\{1\} \times X) .
\end{aligned}
$$

Taking the supremum over all $b \in\{1\} \times X$ yields

$$
\sup _{b \in\{1\} \times X} d(b, A) \leq \sup _{a \in A} d(a,\{1\} \times X),
$$

and the lemma follows.

Next consider a compact connected Riemannian manifold $N$ with connected smooth boundary $\partial N$. Denote by $d$ the distance on $N$ induced by the Riemannian metric. Let $[0,1] \times \partial N$ be a collar neighborhood of $\partial N$ in $N$, where $\partial N$ is identified with $\{1\} \times \partial N$.

Lemma 6. Let $p \in \stackrel{\circ}{N}$ be a given point. Let $\left(A_{n}\right) \subset \stackrel{\circ}{N}$ be a sequence of compact hypersurfaces separating $p$ from $\partial N$ such that

$$
\sup _{a \in A_{n}} d(a, \partial N) \rightarrow 0 \text { as } n \rightarrow \infty \text {. }
$$

Then for $n \in \mathbf{N}$ sufficiently large, $A_{n}$ is contained in the collar neighborhood $[0,1] \times \partial N, A_{n}$ separates $\{0\} \times \partial N$ from $\{1\} \times \partial N$, and

$$
d_{H}\left(A_{n}, \partial N\right) \rightarrow 0 \text { as } n \rightarrow \infty .
$$

Proof. For $n \in \mathbf{N}$ sufficiently large we have $A_{n} \subset(0,1) \times \partial N$. We claim that $A_{n}$ separates $\{0\} \times \partial N$ from $\{1\} \times \partial N$.

By hypothesis, $N \backslash A_{n}$ has precisely 2 connected components $U_{0}^{\prime}, U_{1}$ with $p \in U_{0}^{\prime}$ and $\partial N \subset U_{1}$. Without loss of generality we may assume that $p \notin(0,1] \times \partial N$. Since $N \backslash((0,1] \times \partial N)$ is connected and contains $p$,

$$
N \backslash((0,1] \times \partial N) \subset U_{0}^{\prime} .
$$

Hence $U_{1}$ and $U_{0}:=U_{0}^{\prime} \cap[0,1] \times \partial N$ are disjoint open subsets of $[0,1] \times \partial N$,

$$
\{i\} \times \partial N \subset U_{i} \text { for } i=0,1,
$$

and $U_{1}$ is connected. So the claim is proved if we can show that $U_{0}$ is connected.

Arguing by contradiction, assume that $U_{0}$ is a disjoint union of two open 
subsets $W_{0}$ and $W_{1}$. Since $\{0\} \times \partial N$ is connected, it is contained in one of these sets, say in $W_{0}$. But then

$$
W_{0} \cup[N \backslash((0,1] \times \partial N)]
$$

is open in $U_{0}^{\prime}$, and we have a contradiction to the connectedness of $U_{0}^{\prime}$.

Since the metric induced by $N$ on $[0,1] \times \partial N$ is equivalent to the metric

$$
d^{\prime}((r, x),(s, y))=|r-s|+d(x, y),
$$

Lemma 5 implies

$$
d_{H}\left(A_{n}, \partial N\right) \rightarrow 0 \text { as } n \rightarrow \infty .
$$

\section{Proof of Corollary 1}

Suppose that $S \in \operatorname{Hyp}^{0}\left(\mathbf{R}^{2 n}\right)$ is a hypersurface as in Theorem 1, and $\left(S_{k}\right)_{k \in \mathbf{N}} \subset \operatorname{Hyp}^{0}\left(\mathbf{R}^{2 n}\right)$ is a sequence of hypersurfaces converging to $S$ in the Hausdorff metric. By definition of the Hausdorff metric, the $S_{k}$ are contained in a tubular neighborhood $[-1,1] \times S$ of $S$ for large $k$. The argument in the proof of Lemma 5 shows that $S_{k}$ separates $\{-1\} \times S$ from $\{1\} \times S$. If the tubular neighborhood is sufficiently small this implies

$$
\begin{aligned}
f\left([-4 b,-a] \times S^{1} \times B^{2 n-2}(4 b)\right) & \subset B\left(S_{k}\right), \\
f\left([a, 4 b] \times S^{1} \times B^{2 n-2}(4 b)\right) & \subset U\left(S_{k}\right) .
\end{aligned}
$$

Hence $S_{k}$ is not of restricted contact type by Theorem 1 .

The proof of Corollary 2 is based on the following lemma.

Lemma 7. Let $S \subset(M, \omega)$ and $\tilde{S} \subset(\tilde{M}, \tilde{\omega})$ be hypersurfaces in symplectic manifolds of the same dimension $2 n$ and $P \subset S, \tilde{P} \subset \tilde{S}$ closed characteristics. Then the following two statements are equivalent:

(i) The linear Poincaré maps of $P$ and $\tilde{P}$ are symplectically conjugate.

(ii) There exists a symplectomorphism $F:(U, \omega) \rightarrow(\tilde{U}, \tilde{\omega})$ between tubular neighborhoods of $P, \tilde{P}$ in $M, \tilde{M}$ such that $F(P)=\tilde{P}$, and $F(S \cap U)$ is tangent of second order to $\tilde{S} \cap \tilde{U}$ along $\tilde{P}$.

Remark. Note that the statement is not tautological. For instance, it implies that at a critical point $p$ of a 1-periodic time-dependent Hamiltonian $h$ on a symplectic manifold $(M, \omega)$ the second derivative $h^{\prime \prime}(p)$ can be made time-independent by a 1-periodic time-dependent symplectic change of coordinates (apply the lemma to the hypersurface $\{r=h(t, z)\}$ in the extended phase space $\left.\mathbf{R} \times S^{1} \times M\right)$. 
Proof. 1. Clearly (ii) implies (i). So let us suppose that the linear Poincare maps are conjugate.

A tubular neighborhood of $P$ in $(M, \omega)$ is symplectomorphic to $\left(W, \omega_{2 n}\right)$, where

$$
W=[-a, a] \times S^{1} \times B^{2 n-2}(b)
$$

with coordinates $(r, t, z), z=x+i y \in \mathbf{R}^{2 n-2}=\mathbf{C}^{n-1}$, and

$$
\begin{aligned}
\omega_{2 n} & =d r \wedge d t+\sum_{j=1}^{n-1} d x_{j} \wedge d y_{j} \\
& =d r \wedge d t+\omega_{2 n-2} .
\end{aligned}
$$

The closed characteristic $P$ corresponds to $\{0\} \times S^{1} \times\{0\}^{2 n-2} \in W$. It follows that the hypersurface $S$ corresponds to the graph

$$
S \cap W=\{r+H(t, z)=0\}
$$

of a function $H$ satisfying $H(t, 0)=0$ and $d H(t, 0)=0$ for all $t \in S^{1}$. After a symplectic change of coordinates we may moreover assume that $H(0, z)=0$ for all $z$.

Let $H_{2}(t, z)$ be the part of $H$ quadratic in $z$, extended to $S^{1} \times \mathbf{R}^{2 n-2}$. Replace $S \cap W$ by the hypersurface

$$
S_{2}:=\left\{r+H_{2}(t, z)=0\right\} \subset \hat{W}:=\mathbf{R} \times S^{1} \times \mathbf{R}^{2 n-2}
$$

which is tangent of second order to $S \cap W$ along $P$. Let $\tilde{H}, \tilde{S}_{2}$ etc. be the analogous objects for $\tilde{S}$. We will show that $S_{2}$ can be mapped onto $\tilde{S}_{2}$ by a symplectomorphism of $\hat{W}$, which implies (ii).

The restriction of $\omega_{2 n}$ to $S_{2}$ is given in coordinates $(t, z)$ by

$$
\left.\omega_{2 n}\right|_{S_{2}}=d t \wedge d_{z} H_{2}+\omega_{2 n-2} .
$$

Its kernel is generated by the vector field

$$
X=\frac{\partial}{\partial t}+Z(t, z),
$$

where the time-dependent vector field $Z$ on $\mathbf{R}^{2 n-2}$ is determined by the equation

$$
\begin{aligned}
0 & =i_{X}\left(\left.\omega_{2 n}\right|_{S_{2}}\right) \\
& =d_{z} H_{2}-d_{z} H_{2}(Z) \cdot d t+i_{Z} \omega_{2 n-2}
\end{aligned}
$$

or equivalently

$$
i_{Z} \omega_{2 n-2}+d_{z} H_{2}=0
$$


We see that for every $t \in S^{1}, Z(t, z)$ is linear in $z$. Let $\tilde{Z}$ be the corresponding vector field for $\tilde{S}$. Notice that $Z(0, z)=\tilde{Z}(0, z)=0$ for all $z$ by the assumption $H(0, z)=\tilde{H}(0, z)=0$.

2. Let $\phi_{t}, \tilde{\phi}_{t}: \mathbf{R}^{2 n-2} \rightarrow \mathbf{R}^{2 n-2}$ be the linear flows generated by the timedependent linear vector fields $Z, \tilde{Z}$. The time-1 maps $\phi_{1}, \tilde{\phi}_{1}$ are the linear Poincare maps of $P, \tilde{P}$ in these coordinates. By hypothesis, there exists a linear symplectic map $B: \mathbf{R}^{2 n-2} \rightarrow \mathbf{R}^{2 n-2}$ such that $\tilde{\phi}_{1}=B \phi_{1} B^{-1}$. Apply the symplectomorphism

$$
\hat{B}(r, t, z):=(r, t, B z)
$$

of $\hat{W}$ to $S_{2}$. The kernel of the restriction of $\omega_{2 n}$ to $\hat{B}\left(S_{2}\right)$ is generated by the vector field

$$
\frac{\partial}{\partial t}+B_{*} Z=\frac{\partial}{\partial t}+B Z B^{-1} .
$$

Its time-1 map equals $B \phi_{1} B^{-1}=\tilde{\phi}_{1}$. Thus after this transformation we may assume that

$$
\phi_{1}=\tilde{\phi}_{1} .
$$

3. Define $\Phi: S^{1} \times \mathbf{R}^{2 n-2} \rightarrow S^{1} \times \mathbf{R}^{2 n-2}$ by

$$
\Phi(t, z):=\left(t, \tilde{\phi}_{\iota} \circ \phi_{t}^{-1}(z)\right) .
$$

Notice that $\Phi(1, z)=\left(1, \tilde{\phi}_{1}^{-1} \circ \phi_{1}(z)\right)=(1, z)$ by Step 2 , so $\Phi$ defines a diffeomorphism of $S^{1} \times \mathbf{R}^{2 n-2}$. Moreover, since the flows of $X, \tilde{X}$ preserve $\left.\omega_{2 n}\right|_{S_{2}}$ respectively $\left.\omega_{2 n}\right|_{\tilde{S}_{2}}$, and $X=\tilde{X}$ at $t=0$, we have

$$
\Phi^{*}\left(\left.\omega_{2 n}\right|_{\tilde{S}_{2}}\right)=\left.\omega_{2 n}\right|_{S_{2}} .
$$

4. Define $\Psi: \hat{W} \rightarrow \hat{W}$,

$$
\Psi(r, t, z):=\left(r+H_{2}(t, z)-\tilde{H}_{2}(\Phi(t, z)), \Phi(t, z)\right) .
$$

The diffeomorphism $\Psi$ maps each hypersurface $S^{c}:=\left\{r+H_{2}(t, z)=c\right\}$ onto $\tilde{S}^{c}:=\left\{r+\tilde{H}_{2}(t, z)=c\right\}$. Therefore by Step 3 it satisfies

$$
\begin{aligned}
\left.\left(\Psi^{*} \omega_{2 n}\right)\right|_{S^{c}} & =\Psi^{*}\left(\left.\omega_{2 n}\right|_{\tilde{S}^{c}}\right) \\
& =\Phi^{*}\left(\left.\omega_{2 n}\right|_{\tilde{S}_{2}}\right) \\
& =\left.\omega_{2 n}\right|_{S_{2}} \\
& =\left.\omega_{2 n}\right|_{S^{c}} .
\end{aligned}
$$


Moreover,

$$
\begin{aligned}
i_{\frac{\partial}{\partial r}}\left(\Psi^{*} \omega_{2 n}\right) & =i_{\left(\Psi_{*}^{-1} \frac{\partial}{\partial r}\right)}\left(\Psi^{*} \omega_{2 n}\right) \\
& =\Psi^{*}\left(i_{\frac{\partial}{\partial r}} \omega_{2 n}\right) \\
& =\Psi^{*} d t \\
& =d t \\
& =i_{\frac{\partial}{\partial r}} \omega_{2 n} .
\end{aligned}
$$

Hence $\Psi^{*} \omega_{2 n}=\omega_{2 n}$, i.e. $\Psi$ is a symplectomorphism mapping $S_{2}$ onto $\tilde{S}_{2}$, and the lemma is proved.

\section{Proof of Corollary 2}

Let $x$ be a nondegenerate linearly stable closed characteristic on the hypersurface $S$ whose linear Poincaré map is symplectically conjugate to the diagonal matrix $\operatorname{diag}\left(e^{i \alpha_{1}}, \ldots, e^{i \alpha_{n-1}}\right)$. Let $(U, \omega)$ be a neighborhood of $x$ in $\mathbf{R}^{2 n}$ as constructed at the beginning of Sect. 3, with the same numbers $\alpha_{1}, \ldots, \alpha_{n-1} \in \mathbf{R} \backslash 2 \pi \mathbf{Z}$. By Lemma 7 we can assume that $S \cap U$ is tangent of second order to the hyperplane $\{0\} \times S^{1} \times B^{2 n-2}(4 b)$ along $\{0\} \times S^{1} \times\{0\}^{2 n-2}$. For every sufficiently small $b>0$ we take $a:=\frac{1}{8} \kappa b^{2}$, $\delta:=\frac{1}{2} \kappa b^{2}$, such that the hypotheses of Proposition 1 are satisfied. Since $a$ depends quadratically on $b$ and $S \cap U$ is flat up to second order in $b$, for sufficiently small $b$ we will have

$$
S \cap U \subset(-a, a) \times S^{1} \times B^{2 n-2}(4 b),
$$

thus $S$ satisfies the hypotheses of Theorem 1. It follows from Theorem 1 that $S$ is not of restricted contact type, and the corollary is proved.

\section{Proof of Corollary 3}

Let $S \in H y p^{0}\left(\mathbf{R}^{2 n}\right)$ be as in Corollary 1 or 2 , and suppose that $A_{1} \subset$ $A_{2} \subset \ldots \subset B(S)$ is an exhaustion of the bounded component $B(S)$ by compact sets with smooth boundaries. Passing to connected components, we may assume that the $A_{k}$ are connected. We have $\sup _{a \in \partial A_{k}} d(a, S) \rightarrow 0$ as $k \rightarrow \infty$. Each connected component $S_{k}$ of $\partial A_{k}$ divides $\mathbf{R}^{n}$ into a bounded and an unbounded component, $B\left(S_{k}\right)$ and $U\left(S_{k}\right)$, with $S \subset U\left(S_{k}\right)$. If $A \backslash S_{k} \subset U\left(S_{k}\right)$ we may replace $A_{k}$ by $A_{k} \cup B\left(S_{k}\right)$, thus getting rid of the boundary component $S_{k}$. Since we cannot get rid of all boundary components, we can choose for every $k$ a boundary component $S_{k}$ with $A_{k} \backslash S_{k} \subset B\left(S_{k}\right)$. Then $S_{k}$ separates $0 \in \mathbf{R}^{2 n}$ from $S$ for large $k$. Hence by Lemma $6, d_{H}\left(S_{k}, S\right) \rightarrow 0$ as $k \rightarrow \infty$. So by Corollary $1, S_{k}$ is not of contact type for large $k$.

For the proof of Corollary 4 we need another lemma. 
Lemma 8. If $M$ is an open manifold of dimension $n \geq 1$, then there exists a subset $N \subset M, N \neq M$, with nonempty smooth boundary $\partial N$ such that $N \backslash \partial N$ is diffeomorphic to $M$.

Remark. In general, an open manifold is not diffeomorphic to the interior of a compact manifold with smooth boundary.

Proof. Without loss of generality assume that $M$ is connected. Let $A_{1} \subset$ $A_{2} \subset \ldots \subset M, \cup_{i \in \mathrm{N}_{0}} A_{i}=M$, be an exhaustion of $M$ by compact subsets. Let $\left(x_{i}\right)_{i \in \mathbf{N}_{0}}$ be a sequence such that for every $i$ the points $x_{i}, x_{i+1}, \ldots$ lie in the same path connected component of $M \backslash A_{i}$. Choose a smooth embedded curve $x:[0, \infty) \rightarrow M$ such that $x(i)=x_{i}$ and $x([i, \infty)) \subset M \backslash A_{i}$ for all $i$. The image of $x$ is then a closed submanifold with boundary of $M$. Pick a Riemannian metric on $M$ for which $x$ is a geodesic parametrized by arclength. This can be done by taking the Euclidean metric on a tubular neighborhood $[0, \infty) \times B^{n-1}(1)$ of the image of $x$ and extending it anyhow to $M$. Let $U$ be another tubular neighborhood of $x$ obtained as the image under the exponential map of the subset

$$
\left\{(t, v) \in[0, \infty) \times\left.\mathbf{R}^{n-1}|| v\right|^{2} \leq \rho(t)\right\}
$$

of the normal bundle over $x$, where $\rho:[0, \infty) \rightarrow(0,1)$ is a suitable function. Its boundary in $M$ is given by

$$
\partial U=\left\{(t, v) \in[0, \infty) \times\left.\mathbf{R}^{n-1}|| v\right|^{2}=\rho(t)\right\} \cup\{0\} \times B^{n-1}(\rho(0)) .
$$

Rescaling in the fibre yields a diffeomorphism

$$
U \cong[0, \infty) \times B^{n-1}(1) .
$$

Take a smooth monotone function $\phi:\left[0, \frac{1}{4}\right) \rightarrow[1, \infty)$ with $\phi(0)=1$ and $\phi(r) \rightarrow \infty$ as $r \rightarrow \frac{1}{4}$ (see Fig. 7). Let

$$
R:=\left\{(t, v) \in[0, \infty) \times B^{n-1}(1)|| v \mid<\frac{1}{2}, t>\phi\left(|v|^{2}\right)\right\} .
$$

Via the diffeomorphism above we can view $R$ as a subset of $U$ and thus of $M$. Its boundary in $M$ is given by

$$
\partial R=\left\{(t, v) \in[0, \infty) \times B^{n-1}(1)|| v \mid<\frac{1}{2}, t=\phi\left(|v|^{2}\right)\right\},
$$

due to the choice of $U$. Now $U \backslash \bar{R}$ is diffeomorphic to $U$ by a diffeomorphism which equals the identity near $\partial U$. Hence $N:=M \backslash R$ is the desired subset. 


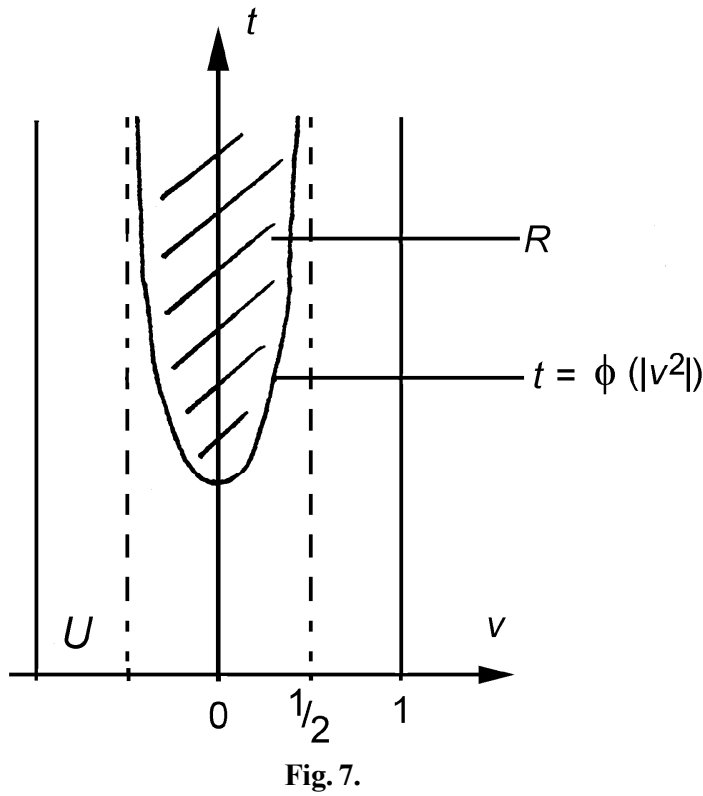

\section{Proof of Corollary 4 (sketch)}

Let $(M, \omega)$ be an open symplectic manifold of dimension $2 n \geq 4$. Let $N \subset$ $M$ be the submanifold provided by Lemma 8 . In a neighborhood of a point of $\partial N$ the triple $(M, N, \omega)$ is symplectomorphic to $\left(\mathbf{R}^{2 n},\{0\} \times \mathbf{R}^{2 n-1}, \omega_{2 n}\right)$ near $0 \in \mathbf{R}^{2 n}$. So (after rescaling) we may replace $\partial N$ in this neighborhood by the hypersurface $f\left(\{0\} \times \mathbf{R}^{2 n-1}\right)$ constructed in Lemma 1 , for some $c<0$. Denote this new hypersurface by $S \subset M$ and its interior by $B(S)$. Note that $B(S)$ is diffeomorphic to $M$.

Now suppose that $(M, \omega)$ is exact convexly exhaustible, and the same is true for $(B(S), \omega)$ (otherwise there is nothing to show). Let $S_{1} \subset B(S)$ be a smooth exact $\omega$-convex compact hypersurface which separates $S$ from the set $f([-4 b,-a] \times V)$ of Lemma 1 , viewed as a subset of $B(S)$. Let $A \subset$ $M$ be a compact subset with smooth $\omega$-convex boundary which contains $f([-4 b, 0] \times V)$ and $S_{1}$. Since $(A, \omega)$ is a compact exact symplectic manifold with $\omega$-convex boundary, we can define the symplectic homology of compact hypersurfaces of $(A, \omega)$, and the analogous statement of Theorem 1 holds. Applied to the hypersurface $S_{1}$ this implies that $S_{1}$ is not of restricted contact type, and we have a contradiction. 


\section{References}

1. K. Cieliebak. Symplectic boundaries: Creating and destroying closed characteristics. To appear in Geom. and Funct. Anal.

2. K. Cieliebak, A. Floer, H. Hofer. Symplectic homology II: A general construction. Math. Zeitschrift 218, 103-122 (1995).

3. K. Cieliebak, A. Floer, H. Hofer, K. Wysocki. Applications of symplectic homology II: Stability of the action spectrum. Math. Zeitschrift 223, 27-45 (1996).

4. Y. Eliashberg, M. Gromov. Convex symplectic manifolds. Proc. of Symposia in Pure Math. 52, Part 2. 135-162 (1991).

5. A. Floer, H. Hofer. Symplectic homology I: Open sets in $\mathbf{C}^{n}$. Math. Zeitschrift 215, 37-88 (1994).

6. A. Floer, H. Hofer, K. Wysocki. Applications of symplectic homology I. Math. Zeitschrift 217, 577-606 (1994).

7. I. Gelfand, V. Lidskij. On the structure of the regions of stability of linear canonical systems of differential equations with periodic coefficients. AMS Translations, Series 2, Vol. 8, 143-181 (1958).

8. M. Gromov. Pseudo holomorphic curves in symplectic manifolds. Invent. math. 82, 307-347 (1985).

9. C. Hummel. Gromov's compactness theorem for pseudo-holomorphic curves. To appear in Birkhäuser-Verlag.

10. D. McDuff. Applications of convex integration to symplectic and contact geometry. Ann. Inst. Fourier Grenoble 37, Part 1. 107-133 (1987).

11. D. Sullivan. Cycles for the dynamical study of foliated manifolds and complex manifolds. Inv. math. 36, 225-255 (1976).

12. A. Weinstein. Symplectic manifolds and their Lagrangian submanifolds. Adv. in Math. 6, 329-346 (1971).

13. A. Weinstein. On the hypotheses of Rabinowitz' periodic orbit theorems. Journ. of Diff. Eq. 33, 353-358 (1979). 